\title{
Access to Endocyclic and Exocyclic Allylic Alcohols by Nickel-Catalyzed Reductive Cyclization of Ynals.
}

\author{
Beth A. Knapp-Reed, Gireesh M. Mahandru, and John Montgomery* \\ Department of Chemistry, Wayne State University, Detroit, MI 48202
}

Unless otherwise noted, all reactions were conducted in flame-dried or oven dried $\left(120^{\circ} \mathrm{C}\right)$ glassware with magnetic stirring under an atmosphere of dry nitrogen or argon. Reaction solvents were purified prior to use. Diethyl ether, tetrahydrofuran, and methylene chloride were purified by passage through a solvent column composed of activated alumina (A-1). ${ }^{1}$ Toluene was dried by distillation from calcium hydride. $N, N$ Dimethylformamide and chloroform were dried over activated $4 \AA$ molecular sieves. Ni $(\mathrm{COD})_{2}$ (Strem Chemicals, Inc.), 1,3-bis(2,4,6-trimethyl-phenyl)imidazolium chloride, 1,3-bis(2,6-diisopropyl-phenyl)imidazolium chloride and $\mathrm{KO}$-Bu (Aldrich) were stored and weighed in an inert atmosphere glovebox. Tributylphosphine (Aldrich) and trimethylphosphine (Strem Chemicals, Inc.) were distilled just prior to use.

Analytical thin layer chromatography (TLC) was performed on Kieselgel 60 F254 (250 $\mu \mathrm{m}$ silica gel) glass plates or aluminum oxide 60 F254 (250 $\mu \mathrm{m})$ alumina plates and compounds were visualized with UV light, iodine, $p$-anisaldehyde stain, ceric ammonium molybdate stain, or aqueous $\mathrm{KMnO}_{4}$ solution. Flash column chromatography was performed using Kieselgel 60 (230-400 mesh) silica gel. Eluent mixtures are reported as $\mathrm{v}: \mathrm{v}$ percentages of the minor constituent in the major constituent and correspond to $\mathrm{R}_{\mathrm{f}}$ values between 0.2 and 0.3 . All compounds purified by column chromatography were sufficiently pure for use in further experiments unless otherwise indicated.

${ }^{1} \mathrm{H}$ NMR spectra were measured at $400 \mathrm{MHz}$ on a Varian Mercury 400 instrument or at $500 \mathrm{MHz}$ on a Varian Unity 500 instrument. The proton signal of the residual, nondeuterated solvent $\left(\delta 7.26\right.$ for $\mathrm{CHCl}_{3}$ ) was used as an internal reference for ${ }^{1} \mathrm{H}$ NMR spectra. ${ }^{13} \mathrm{C}$ NMR spectra were completely heterodecoupled and measured at 125 or 100 MHz. Residual chloroform ( $\delta 77.0)$ was used as an internal reference. High resolution 
mass spectra were recorded on a VG 70-250-s spectrometer manufactured by Micromass Corp. (Manchester UK) at the University of Michigan Mass Spectrometry Laboratory.

\section{General procedure A- carbene-mediated ynal cyclizations.}

To a solution of $\mathrm{Ni}(\mathrm{COD})_{2}(20 \mathrm{mmol} \%)$, imidazolium salt 2 or 3 (20 mol\%), and $\mathrm{KO} t$ $\mathrm{Bu}(20 \mathrm{~mol} \%)$ in toluene $(0.006 \mathrm{M})$ was added $\mathrm{Et}_{3} \mathrm{SiH}(5 \mathrm{eq}$.$) . The resulting mixture was$ heated to $75{ }^{\circ} \mathrm{C}$. After $15 \mathrm{~min}$ the aldehyde (1 eq.) was added dropwise. The reaction mixture was heated at $75{ }^{\circ} \mathrm{C}$ for an additional $1 \mathrm{~h}$. The solution was cooled to $\mathrm{rt}$, the solvent removed in vacuo, and the residue purified via column chromatography to afford the desired macrocycles.

\section{General procedure B- phosphine-mediated ynal cyclizations. ${ }^{2}$}

To a solution of $\mathrm{Ni}(\mathrm{COD})_{2}(20 \mathrm{~mol} \%)$ in toluene $(0.006 \mathrm{M}), \mathrm{Bu}_{3} \mathrm{P}$ or $\mathrm{Me}_{3} \mathrm{P}(40 \mathrm{~mol} \%)$ and $\mathrm{Et}_{3} \mathrm{~B}$ (4 eq.) were added. The resulting mixture was heated to $60{ }^{\circ} \mathrm{C}$ for $5 \mathrm{~min}$. The aldehyde (1 eq.) was then added as a toluene solution. The reaction mixture was then stirred overnight at $60{ }^{\circ} \mathrm{C}$. The solution was then cooled to $\mathrm{rt}$, the solvent removed in vacuo, and the residue purified via column chromatography to give the desired macrocycle.

\section{(Cycloundec-2(E)-enyloxy)-triethylsilane (4b)}

OTES Following general procedure $\mathrm{A}, \mathrm{Ni}(\mathrm{COD})_{2}(0.017 \mathrm{~g}, 0.060 \mathrm{mmol})$, imidazolium

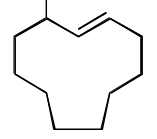

salt 2 (0.020 g, $0.060 \mathrm{mmol}), \mathrm{KO} t$-Bu (0.007 g, $0.06 \mathrm{mmol})$, triethylsilane $(0.24$ $\mathrm{mL}, 1.5 \mathrm{mmol})$ and undec-10-ynal $(0.050 \mathrm{~g}, 0.30 \mathrm{mmol})$ gave, after column chromatography (5\% ether/hexanes), $\mathbf{4 b}$ as a light-colored semi-solid $(0.042 \mathrm{~g}, 50 \%) .{ }^{1} \mathrm{H}$ NMR $\left(\mathrm{CDCl}_{3}, 400 \mathrm{MHz}\right) \delta 5.56(\mathrm{ddd}, J=5.6,9.6,14.8 \mathrm{~Hz}, 1 \mathrm{H}), 5.38(\mathrm{dd}, J=8.0,15.6$ $\mathrm{Hz}, 1 \mathrm{H}), 4.00$ (dt, $J=4.0,9.2 \mathrm{~Hz}, 1 \mathrm{H}), 2.21-2.15$ (m, 1H), 2.05-1.96 (m, 1H), 1.77-1.70 (m, 1H), 1.64-1.59 (m, 1H), 1.50-1.09 (m, 11H), 1.07-0.98 (m, 1H), 0.93 (t, $J=8.1 \mathrm{~Hz}$, 9H), 0.57 (q, $J=8.1 \mathrm{~Hz}, 6 \mathrm{H}) ;{ }^{13} \mathrm{C} \mathrm{NMR}\left(\mathrm{CDCl}_{3}, 100 \mathrm{MHz}\right) \delta 134.3,130.7,75.6,36.7$, $34.2,26.7,26.34,26.3,25.6,20.8,7.1,5.1$; IR (thin film) v 3405, 2930, 1461, 1064, 726 $\mathrm{cm}^{-1}$; HRMS (ES+) calcd. for $\mathrm{C}_{17} \mathrm{H}_{34} \mathrm{OSi}[\mathrm{M}]^{+} 282.2379$, found: 282.2377. 


\section{(Cyclotetradec-2(E)-enyloxy)-triethylsilane (4c).}

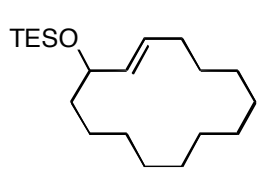

Following general procedure $\mathrm{A}, \mathrm{Ni}(\mathrm{COD})_{2}(0.013 \mathrm{~g}, 0.048 \mathrm{mmol})$, imidazolium salt 2 (0.016 g, $0.048 \mathrm{mmol}), \mathrm{KO} t$-Bu $(0.005 \mathrm{~g}, 0.05$ $\mathrm{mmol})$, triethylsilane $(0.19 \mathrm{~mL}, 1.2 \mathrm{mmol})$ and tetradec-13-ynal $(0.050$ g, $0.24 \mathrm{mmol}$ ) gave, after column chromatography (5\% ether/hexanes), $4 \mathbf{c}$ as a white semi-solid (0.048 g, 62\%). ${ }^{1} \mathrm{H} \mathrm{NMR}\left(\mathrm{CDCl}_{3}, 400 \mathrm{MHz}\right) \delta 5.40(\mathrm{ddd}, J=15.4,8.9,4.1$ $\mathrm{Hz}, 1 \mathrm{H}), 5.32$ (dd, $J=7.3,15.4 \mathrm{~Hz}, 1 \mathrm{H}), 4.10(\mathrm{ddd}, J=3.2,7.3,10.5 \mathrm{~Hz}, 1 \mathrm{H}), 2.20-2.14$ (m, 1H), 2.01-1.92 (m, 1H), 1.57-1.21 (m, 20H), 0.94 (t, $J=8.1 \mathrm{~Hz}, 9 \mathrm{H}), 0.57$ (q, $J=8.1$ $\mathrm{Hz}, 6 \mathrm{H}) ;{ }^{13} \mathrm{C} \mathrm{NMR}\left(\mathrm{CDCl}_{3}, 100 \mathrm{MHz}\right) \delta 134.8,131.4,74.0,37.1,31.2,27.3,26.5,26.1$, $25.2,25.0,24.3,23.4,23.3,21.8,6.8,4.9$; IR (thin film) v 2929, 2857, 1710, 1458, 1413, 1349, 1238, 1070, 1006, 971, $743 \mathrm{~cm}^{-1}$; GCMS calcd. for $\mathrm{C}_{20} \mathrm{H}_{40} \mathrm{OSi}[\mathrm{M}]^{+}$324, found: 324.

\section{1-Triethylsilanyloxy-oxacyclopentadec-12(E)-en-2-one (4d).}

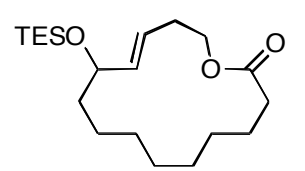

Following general procedure $\mathrm{A}, \mathrm{Ni}(\mathrm{COD})_{2}(0.011 \mathrm{~g}, 0.040 \mathrm{mmol})$, imidazolium salt $2(0.014 \mathrm{~g}, 0.040 \mathrm{mmol}), \mathrm{KO} t$-Bu $(0.004 \mathrm{~g}, 0.04$ mmol), triethylsilane $(0.17 \mathrm{~mL}, 1.0 \mathrm{mmol})$ and aldehyde $\mathbf{1 d}(0.049 \mathrm{~g}$, $0.21 \mathrm{mmol})$ gave, after column chromatography (10\% ether/hexanes), $4 \mathbf{d}$ as a colorless oil $(0.050 \mathrm{~g}, 69 \%)$. ${ }^{1} \mathrm{H}$ NMR $\left(\mathrm{CDCl}_{3}, 400 \mathrm{MHz}\right) \delta 5.54(\mathrm{~m}, 2 \mathrm{H}), 4.23$ (ddd, $J=4.9,7.3$, $11.4 \mathrm{~Hz}, 1 \mathrm{H}), 4.10-4.02(\mathrm{~m}, 2 \mathrm{H}), 2.40$ (m, 2H), 2.33 (ddd, $J=4.9,7.3,9.7 \mathrm{~Hz}, 2 \mathrm{H}), 1.69-$ $1.47(\mathrm{~m}, 4 \mathrm{H}), 1.37-1.18(\mathrm{~m}, 10 \mathrm{H}), 0.94(\mathrm{t}, J=8.1 \mathrm{~Hz}, 9 \mathrm{H}), 0.57(\mathrm{q}, J=8.1 \mathrm{~Hz}, 6 \mathrm{H}) ;{ }^{13} \mathrm{C}$ NMR $\left(\mathrm{CDCl}_{3}, 100 \mathrm{MHz}\right) \delta$ 174.4, 136.0, 127.6, 73.4, 63.9, 37.5, 33.4, 31.0, 27.0, 26.9, 26.5, 25.8, 24.8, 22.7, 6.8, 4.9; IR (thin film) v 2933, 2875, 2859, 1735, 1458, 1236, 1173, 1149, 1057, 1005, 968, $743 \mathrm{~cm}^{-1}$; HRMS (ES+) calcd. for $\mathrm{C}_{20} \mathrm{H}_{38} \mathrm{O}_{3} \mathrm{SiNa}[\mathrm{M}+\mathrm{Na}]^{+}$ 377.2488, found: 377.2484 .

\section{1-Triethylsilanyloxy-oxacyclopentadec-12(E)-en-2-one (4e).}

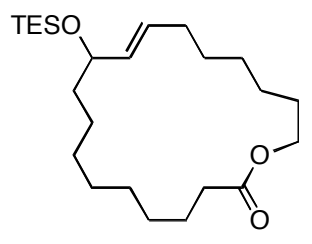

Following general procedure $\mathrm{A}, \mathrm{Ni}(\mathrm{COD})_{2}(0.017 \mathrm{~g}, 0.060 \mathrm{mmol})$, imidazolium salt 2 (0.020 g, $0.060 \mathrm{mmol}), \mathrm{KO} t-\mathrm{Bu}(0.007 \mathrm{~g}, 0.06$ $\mathrm{mmol})$, triethylsilane $(0.24 \mathrm{~mL}, 1.5 \mathrm{mmol})$ and aldehyde $1 \mathrm{e}(0.088 \mathrm{~g}$, $0.30 \mathrm{mmol})$ gave, after column chromatography (10\% 
ether/hexanes), $4 \mathbf{e}$ as a colorless oil $(0.086 \mathrm{~g}, 70 \%) .{ }^{1} \mathrm{H} \mathrm{NMR}\left(\mathrm{CDCl}_{3}, 500 \mathrm{MHz}\right) \delta 5.48$ (ddd, $J=5.7,8.1,15.4 \mathrm{~Hz}, 1 \mathrm{H}), 5.34(\mathrm{dd}, J=7.3,15.4 \mathrm{~Hz}, 1 \mathrm{H}), 4.09$ (m, 2H), 4.00 (m, 1H), 2.31 (app. t, $J=6.5 \mathrm{~Hz}, 2 \mathrm{H}), 2.09-1.94(\mathrm{~m}, 2 \mathrm{H}), 1.67-1.53(\mathrm{~m}, 4 \mathrm{H}), 1.48-1.21(\mathrm{~m}$, $18 \mathrm{H}), 0.93(\mathrm{t}, J=8.1 \mathrm{~Hz}, 9 \mathrm{H}), 0.57(\mathrm{q}, J=8.1 \mathrm{~Hz}, 6 \mathrm{H}) ;{ }^{13} \mathrm{C} \mathrm{NMR}\left(\mathrm{CDCl}_{3}, 125 \mathrm{MHz}\right) \delta$ 173.9, 134.1, 130.7, 73.9, 63.9, 49.6, 37.9, 34.6, 31.5, 29.3, 29.2, 28.7, 28.6, 28.2, 27.8, 25.9, 24.9, 24.7, 6.8, 4.9; IR (thin film) v 2930, 2855, 1736, 1237, 1063, 1005, 970, 743 $\mathrm{cm}^{-1}$; HRMS (ES+) calcd. for $\mathrm{C}_{24} \mathrm{H}_{46} \mathrm{O}_{3} \mathrm{SiNa}[\mathrm{M}+\mathrm{Na}]^{+}$433.3114, found: 433.3108 .

\section{1-Triethylsilanyloxy-oxacyclodocos-12(E)-en-2-one (4f).}

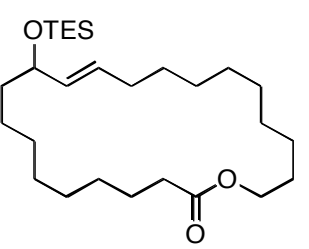

Following general procedure $\mathrm{A}, \mathrm{Ni}(\mathrm{COD})_{2}(0.008 \mathrm{~g}, 0.03 \mathrm{mmol})$, imidazolium salt $2(0.010 \mathrm{~g}, 0.030 \mathrm{mmol}), \mathrm{KO} t$-Bu $(0.004 \mathrm{~g}, 0.03$ $\mathrm{mmol})$, triethylsilane $(0.12 \mathrm{~mL}, 0.74 \mathrm{mmol})$ and aldehyde $\mathbf{1 f}(0.050$ $\mathrm{g}, \quad 0.15 \mathrm{mmol})$ gave, after column chromatography $(10 \%$ ether/hexanes), $\mathbf{4 f}$ as a colorless oil $(0.044 \mathrm{~g}, 67 \%) .{ }^{1} \mathrm{H} \mathrm{NMR}\left(\mathrm{CDCl}_{3}, 400 \mathrm{MHz}\right) \delta 5.42$ (ddd, $J=4.9,8.1,15.4 \mathrm{~Hz}, 1 \mathrm{H}), 5.30(\mathrm{dd}, J=7.3,15.4 \mathrm{~Hz}, 1 \mathrm{H}), 4.10(\mathrm{dt}, J=2.4,6.5 \mathrm{~Hz}$, $2 \mathrm{H}$ ), $3.98(\mathrm{dt}, J=4.9,8.1 \mathrm{~Hz}, 1 \mathrm{H}), 2.29(\mathrm{t}, J=7.3 \mathrm{~Hz}, 2 \mathrm{H}), 2.10-2.04(\mathrm{~m}, 1 \mathrm{H}), 1.99-1.90$ (m, 1H), 1.61-1.51 (m, 4H), 1.45-1.25 (m, 24H), 0.93 (t, $J=8.1 \mathrm{~Hz}, 9 \mathrm{H}), 0.56$ (q, $J=8.1$ $\mathrm{Hz}, 6 \mathrm{H}) ;{ }^{13} \mathrm{C} \mathrm{NMR}\left(\mathrm{CDCl}_{3}, 100 \mathrm{MHz}\right) \delta 173.9,134.1,130.9,74.0,63.9,38.4,34.9,31.7$, 29.7, 29.6, 29.2, 29.1, 28.9, 28.7, 28.5, 28.3, 26.0, 25.5, 25.3, 6.8, 4.9; IR (thin film) $v$ 2927, 2854, 2874, 1736, 1463, 1237, 1154, 1066, 1005, 970, $744 \mathrm{~cm}^{-1}$; HRMS (ES+) calcd. for $\mathrm{C}_{27} \mathrm{H}_{52} \mathrm{O}_{3} \mathrm{SiNa}[\mathrm{M}+\mathrm{Na}]^{+}$475.3583, found: 475.3687 .

\section{3,12-Bis-triethylsilanyloxy-cyclooctadeca-1,10(E,E)-diene (5a).}

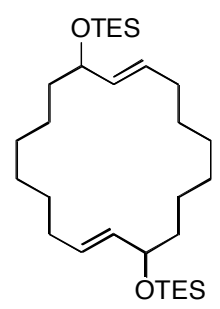

Following general procedure $\mathrm{A}, \mathrm{Ni}(\mathrm{COD})_{2}(0.020 \mathrm{~g}, 0.072 \mathrm{mmol})$, imidazolium salt $2(0.028 \mathrm{~g}, 0.072 \mathrm{mmol}), \mathrm{KO} t-\mathrm{Bu}(0.008 \mathrm{~g}, 0.07 \mathrm{mmol})$, triethylsilane $(0.29 \mathrm{~mL}, 1.8 \mathrm{mmol})$ and non-8-ynal $(0.050 \mathrm{~g}, 0.30 \mathrm{mmol})$ gave, after column chromatography (5\% ether/hexanes), $\mathbf{5 a}$ as a mixture of inseparable diastereomers $(0.030 \mathrm{~g}, 33 \%)$. ${ }^{1} \mathrm{H} \mathrm{NMR}\left(\mathrm{CDCl}_{3}, 500 \mathrm{MHz}\right) \delta$

5.37 (ddd, $J=5.6 \mathrm{~Hz}, J=5.6,9.1,15.0 \mathrm{~Hz}, 2 \mathrm{H}), 5.27(\mathrm{dd}, J=7.0 \mathrm{~Hz}, 15.0 \mathrm{~Hz}, 2 \mathrm{H}$ ), 4.02-3.94 (m, 2H), 2.15-2.06 (m, 2H), 2.00-1.85 (m, 2H), 1.57-1.15 (m, 20H), 0.94 (t, $J=$ $8.1 \mathrm{~Hz}, 18 \mathrm{H}), 0.57(\mathrm{q}, J=8.1 \mathrm{~Hz}, 12 \mathrm{H}) ;{ }^{13} \mathrm{C} \mathrm{NMR}\left(\mathrm{CDCl}_{3}, 100 \mathrm{MHz}\right) \delta 134.3,131.3$, 
130.9, 74.4, 73.9, 38.4, 38.2, 32.1, 31.8, 29.5, 29.1, 28.7, 28.5, 26.0, 25.6, 6.8, 4.9; IR (thin film) $v 2926,2854,1460,1237,1068,1005,968,743 \mathrm{~cm}^{-1}$; HRMS (ES+) calcd. for $\mathrm{C}_{30} \mathrm{H}_{60} \mathrm{O}_{2} \mathrm{Si}_{2} \mathrm{Na}[\mathrm{M}+\mathrm{Na}]^{+}$531.4030, found: 531.4037 .

12-Methyl-11-triethylsilanyloxy-oxacyclodocos-12-en-2-one (7c) and 12-Ethylidene-

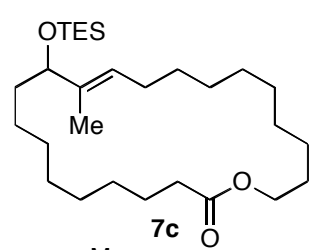
11-triethylsilanyloxy-oxacycloheneicosan-2-one (8c) - Table 2, Entry 3.

Following general procedure $\mathrm{A}, \mathrm{Ni}(\mathrm{COD})_{2}(0.007 \mathrm{~g}, 0.03 \mathrm{mmol})$, imidazolium salt $2(0.009 \mathrm{~g}, 0.03 \mathrm{mmol}), \mathrm{KO} t$-Bu $(0.003 \mathrm{~g}, 0.03$

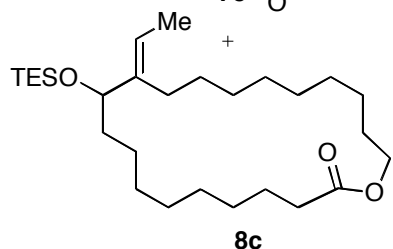
$\mathrm{mmol})$, triethylsilane $(0.10 \mathrm{~mL}, 0.65 \mathrm{mmol})$ and aldehyde $\mathbf{6 b}$ $(0.045 \mathrm{~g}, 0.13 \mathrm{mmol})$ gave, after column chromatography (10\% ether/hexanes), 7c and 8c as an inseparable 1:1 mixture of isomers $(0.036 \mathrm{~g}, 60 \%)$. ${ }^{1} \mathrm{H}$ NMR peaks for $7 \mathbf{c}\left(\mathrm{CDCl}_{3}, 500 \mathrm{MHz}\right) \delta 5.22(\mathrm{dd}, J=6.1,8.6$ Hz, 1H), 4.14-4.07 (m, 2H), 3.94 (dd, $J=9.1,5.1 \mathrm{~Hz}, 1 \mathrm{H}), 2.30$ (t, $J=7.1 \mathrm{~Hz}, 2 \mathrm{H}), 2.14-$ $2.06(\mathrm{~m}, 1 \mathrm{H}), 1.97-1.87(\mathrm{~m}, 1 \mathrm{H}), 1.65-1.56(\mathrm{~m}, 7 \mathrm{H}), 1.54-1.42(\mathrm{~m}, 2 \mathrm{H}), 1.39-1.17(\mathrm{~m}$, $22 \mathrm{H}), 0.92(\mathrm{t}, J=8.1 \mathrm{~Hz}, 6 \mathrm{H}), 0.55(\mathrm{q}, J=8.1 \mathrm{~Hz}, 9 \mathrm{H}) ;{ }^{1} \mathrm{H}$ NMR peaks for $8 \mathrm{c}\left(\mathrm{CDCl}_{3}\right.$, $500 \mathrm{MHz}) \delta 5.34(\mathrm{q}, J=7.1 \mathrm{~Hz}, 1 \mathrm{H}), 4.14-4.07(\mathrm{~m}, 2 \mathrm{H}), 3.89(\mathrm{t}, J=6.6 \mathrm{~Hz}, 1 \mathrm{H}), 2.30(\mathrm{t}$, $J=6.6 \mathrm{~Hz}, 2 \mathrm{H}), 2.14-2.06(\mathrm{~m}, 1 \mathrm{H}), 1.97-1.87(\mathrm{~m}, 1 \mathrm{H}), 1.65-1.56(\mathrm{~m}, 7 \mathrm{H}), 1.54-1.42(\mathrm{~m}$, $2 \mathrm{H}), 1.39-1.17(\mathrm{~m}, 22 \mathrm{H}), 0.91(\mathrm{t}, J=8.1 \mathrm{~Hz}, 6 \mathrm{H}), 0.54(\mathrm{q}, J=8.1 \mathrm{~Hz}, 9 \mathrm{H}) ;{ }^{13} \mathrm{C}$ NMR of 7c/8c mixture $\left(\mathrm{CDCl}_{3}, 125 \mathrm{MHz}\right) \delta 173.9,136.9,126.4,121.0,94.8,78.8,78.6,64.2$, 63.9, 36.7, 35.8, 35.1, 34.8, 30.0, 29.7, 29.6, 29.5, 29.4, 29.3, 29.2, 29.1, 29.0, 28.9, 28.7, 28.5, 27.0, 26.5, 26.2, 25.9, 25.7, 25.6, 25.4, 13.1, 10.5, 6.8, 4.8; IR of 7c/8c mixture $\left(\right.$ thin film) $v 2928,2854,2874,1736,1460,1237,1065,1005,724 \mathrm{~cm}^{-1}$; HRMS (ES+) calcd. for $\mathrm{C}_{28} \mathrm{H}_{54} \mathrm{O}_{3} \mathrm{SiNa}[\mathrm{M}+\mathrm{Na}]^{+} 489.3740$, found: 489.3752 .

To a solution of 7c and $8 \mathbf{c}(0.020 \mathrm{~g}, 0.043 \mathrm{mmol})$ in THF $(0.22 \mathrm{~mL})$ at $0{ }^{\circ} \mathrm{C}$, TBAF $(0.064 \mathrm{~mL}$ of a $1.0 \mathrm{M}$ solution in THF) was added. The resulting solution was stirred at $0{ }^{\circ} \mathrm{C}$ for $2 \mathrm{~h}$. The reaction mixture was then concentrated and purified via column chromatography (20\% ethyl acetate/hexanes) to give $0.006 \mathrm{~g}$ of $\mathbf{7 d}$ and $0.007 \mathrm{~g}$ 8d. These compounds were spectroscopically identical to those obtained from $\mathbf{6 b}$ using general procedure $\mathrm{B}$. 


\section{Table 2, Entry 4.}

Following general procedure $\mathrm{A}, \mathrm{Ni}(\mathrm{COD})_{2}(0.013 \mathrm{~g}, 0.046 \mathrm{mmol})$, imidazolium salt 3 (0.019 g, $0.046 \mathrm{mmol}), \mathrm{KO} t-\mathrm{Bu}(0.005 \mathrm{~g}, 0.05 \mathrm{mmol})$, triethylsilane $(0.180 \mathrm{~mL}, 1.2$ mmol) and aldehyde $\mathbf{6 b}(0.080 \mathrm{~g}, 0.23 \mathrm{mmol})$ gave, after column chromatography (10\% ether/hexanes), 7c and 8c as an inseparable 1:5 mixture of isomers (0.099 $\mathrm{g}, 93 \%)$.

\section{1-Hydroxy-12-methyl-oxacyclodocos-12-en-2-one (7d) and 12-Ethylidene-11-} hydroxy-oxacycloheneicosan-2-one $(8 d)$.

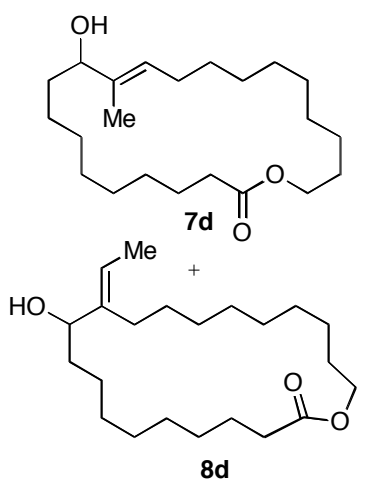

Following general procedure $\mathrm{B}, \mathrm{Ni}(\mathrm{COD})_{2}(0.009 \mathrm{~g}, 0.03 \mathrm{mmol})$, $\mathrm{Me}_{3} \mathrm{P}(0.006 \mathrm{~mL}, 0.06 \mathrm{mmol})$, triethylborane $(0.63 \mathrm{~mL}$ of a $1.0 \mathrm{M}$ solution in toluene) and aldehyde $6 \mathbf{a}(0.055 \mathrm{~g}, 0.16 \mathrm{mmol})$ gave, after column chromatography (25\% ethyl acetate/hexanes), a mixture of $7 \mathbf{d}$ and $\mathbf{8 d}(0.049 \mathrm{~g}, 89 \%)$. The alcohols could be separated after a careful column (20\% ethyl acetate/hexanes) to give $0.039 \mathrm{~g}$ of $\mathbf{7 d}$ and $0.08 \mathrm{~g}$ of $\mathbf{8 d}$. Data for $\mathbf{7 d}$ : ${ }^{1} \mathrm{H} \mathrm{NMR}\left(\mathrm{CDCl}_{3}\right.$, $400 \mathrm{MHz}) \delta 5.32$ (app. t, $J=7.3 \mathrm{~Hz}, 1 \mathrm{H}), 4.10(\mathrm{dt}, J=6.5,1.6 \mathrm{~Hz}, 2 \mathrm{H}), 4.00(\mathrm{t}, J=7.3$ $\mathrm{Hz}, 1 \mathrm{H}), 2.29$ (t, $J=7.3 \mathrm{~Hz}, 2 \mathrm{H}), 2.16-2.07$ (m, 1H), 2.01-1.93 (m, 1H), 1.62-1.51 (m, $10 \mathrm{H}), 1.36-1.10(\mathrm{~m}, 22 \mathrm{H}) ;{ }^{13} \mathrm{C} \mathrm{NMR}\left(\mathrm{CDCl}_{3}, 100 \mathrm{MHz}\right) \delta 173.9,136.4,127.8,78.2$, 63.9, 35.0, 34.2, 30.0, 29.7, 29.6, 29.4, 29.3, 29.2 , 28.8, 28.6, 28.5, 27.0, 25.9, 25.6, 25.5, 10.6; IR (thin film) v 3316, 2923, 2850, 1729, 1456, 1339, 1251, 1164, 1103, 1063, 1013 $\mathrm{cm}^{-1}$; HRMS (ES+) calcd. for $\mathrm{C}_{22} \mathrm{H}_{40} \mathrm{O}_{3} \mathrm{Na}[\mathrm{M}+\mathrm{Na}]^{+}$375.2875, found: 375.2865. Data for 8d: ${ }^{1} \mathrm{H} \mathrm{NMR}\left(\mathrm{CDCl}_{3}, 400 \mathrm{MHz}\right) \delta 5.47(\mathrm{q}, J=6.5 \mathrm{~Hz}, 1 \mathrm{H}), 4.11(\mathrm{dt}, J=5.6,1.6 \mathrm{~Hz}$, 2H), $3.96(\mathrm{t}, J=6.5 \mathrm{~Hz}, 1 \mathrm{H}), 2.31(\mathrm{t}, J=6.5 \mathrm{~Hz}, 2 \mathrm{H}), 2.13-2.07(\mathrm{~m}, 1 \mathrm{H}), 1.99-1.95(\mathrm{~m}$, $1 \mathrm{H}), 1.63-1.48(\mathrm{~m}, 10 \mathrm{H}), 1.40-1.28(\mathrm{~m}, 22 \mathrm{H})$; ${ }^{13} \mathrm{C} \mathrm{NMR}\left(\mathrm{CDCl}_{3}, 100 \mathrm{MHz}\right) \delta 174.0$, $142.5,122.0,77.8,64.2,35.2,34.7,29.7,29.5,29.4,29.2,29.1,29.0,28.9,28.8,28.7$, 26.6, 26.2, 26.2, 25.7, 25.3, 13.2; IR (thin film) v 3423, 2926, 2854, 1734, 1463, 1384, 1236, 1178. 1103, $1039 \mathrm{~cm}^{-1}$; HRMS (ES+) calcd. for $\mathrm{C}_{22} \mathrm{H}_{40} \mathrm{O}_{3} \mathrm{Na}[\mathrm{M}+\mathrm{Na}]^{+} 375.2875$, found: 375.2865 . 


\section{2-Benzylidene-11-triethylsilanyloxy-oxacycloheneicosan-2-one (8a).}

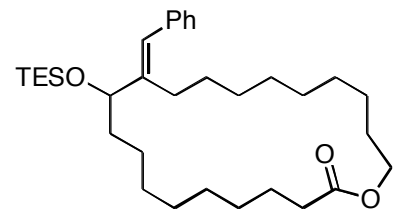

Following general procedure $\mathrm{A}, \mathrm{Ni}(\mathrm{COD})_{2}(0.011 \mathrm{~g}, 0.039$ mmol), imidazolium salt $2(0.013 \mathrm{~g}, 0.039 \mathrm{mmol}), \mathrm{KO} t$ - $\mathrm{Bu}$

$(0.004 \mathrm{~g}, 0.04 \mathrm{mmol})$, triethylsilane $(0.16 \mathrm{~mL}, 0.97 \mathrm{mmol})$ and aldehyde 6a $(0.080 \mathrm{~g}, 0.19 \mathrm{mmol})$ gave, after column chromatography (10\% ether/hexanes), 8a as a colorless oil $(0.070 \mathrm{~g}, 68 \%) .{ }^{1} \mathrm{H}$ NMR $\left(\mathrm{CDCl}_{3}, 400 \mathrm{MHz}\right) \delta$ 7.34$7.30(\mathrm{~m}, 2 \mathrm{H}), 7.25-7.18(\mathrm{~m}, 3 \mathrm{H}), 6.42(\mathrm{~s}, 1 \mathrm{H}), 4.12-4.09(\mathrm{~m}, 3 \mathrm{H}), 2.33-2.15(\mathrm{~m}, 4 \mathrm{H})$, $1.66-1.60(\mathrm{~m}, 6 \mathrm{H}), 1.46-1.25(\mathrm{~m}, 24 \mathrm{H}), 0.96(\mathrm{t}, J=8.1 \mathrm{~Hz}, 9 \mathrm{H}), 0.60(\mathrm{q}, J=8.1 \mathrm{~Hz}, 6 \mathrm{H})$; ${ }^{13} \mathrm{C} \mathrm{NMR}\left(\mathrm{CDCl}_{3}, 100 \mathrm{MHz}\right) \delta 174.1,145.3,138.2,128.6,128.1,126.1,125.7,78.0$, 64.2, 36.8, 34.8, 29.8, 29.5, 29.2, 29.0, 28.9, 28.8, 28.7, 28.6, 28.4, 28.0, 26.1, 25.5, 25.4, 7.0, 4.9; IR (thin film) $v$ 2928, 2874, 2854, 1735, 1460, 1237, 1175, 1070, 1005, 743, $698 \mathrm{~cm}^{-1}$; HRMS (ES+) calcd. for $\mathrm{C}_{33} \mathrm{H}_{56} \mathrm{O}_{3} \mathrm{SiNa}[\mathrm{M}+\mathrm{Na}]^{+}$551.3896, found: 551.3895 .

\section{2-Benzylidene-11-hydroxy-oxacycloheneicosan-2-one (8b).}

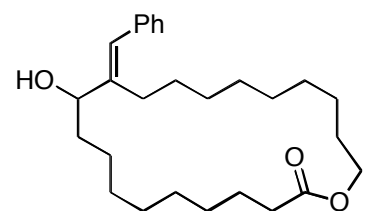

Following general procedure $\mathrm{B}, \mathrm{Ni}(\mathrm{COD})_{2}(0.007 \mathrm{~g}, 0.03 \mathrm{mmol})$, $\mathrm{Bu}_{3} \mathrm{P}(0.012 \mathrm{~mL}, 0.048 \mathrm{mmol})$, triethylborane $(0.070 \mathrm{~mL}, 0.49$ $\mathrm{mmol})$ and aldehyde $\mathbf{6 a}(0.050 \mathrm{~g}, 0.12 \mathrm{mmol})$ gave, after column chromatography (10\% ethyl acetate/hexanes), $\mathbf{8 b}$ as a colorless oil (0.024 g, 48\%). (A small amount (8\%) of an isomer of undetermined structure was present by ${ }^{1} \mathrm{H}$ NMR. It is possible that this is the endocyclic isomer.) ${ }^{1} \mathrm{H} \mathrm{NMR}\left(\mathrm{CDCl}_{3}, 400 \mathrm{MHz}\right) \delta$ 7.37-7.31 (m, 2H), 7.28-7.19 (m, 3H), $6.5(\mathrm{~s}, 1 \mathrm{H}), 4.18(\mathrm{t}, J=6.8 \mathrm{~Hz}, 1 \mathrm{H}), 4.11(\mathrm{t}, J=5.6 \mathrm{~Hz}, 2 \mathrm{H})$, $2.31(\mathrm{t}, J=7.2 \mathrm{~Hz}, 2 \mathrm{H}), 1.66-1.58(\mathrm{~m}, 7 \mathrm{H}), 1.49-1.43(\mathrm{~m}, 3 \mathrm{H}), 1.35-1.25(\mathrm{~m}, 21 \mathrm{H}) ;{ }^{13} \mathrm{C}$ NMR $\left(\mathrm{CDCl}_{3}, 100 \mathrm{MHz}\right) \delta$ 174.3, 145.5, 137.9, 128.9, 128.4, 126.7, 126.3, 77.28, 64.4, 35.6, 35.0, 29.8, 29.6, 29.4, 29.3, 29.2, 29.1, 28.9, 28.8, 28.6, 28.3, 26.3, 25.8, 25.5; IR (thin film) $3416,2926,2853,1733,1463,698 \mathrm{~cm}^{-1}$; HRMS (EI) calcd. for $\mathrm{C}_{27} \mathrm{H}_{42} \mathrm{O}_{3}[\mathrm{M}]^{+}$ 414.3143, found: 414.3128 . 


\section{Starting Materials}

Non-8-ynal (1a), ${ }^{3}$ undec-10-ynal (1b), ${ }^{3}$ and tetradec-13-ynal (1c) ${ }^{4}$ were all prepared according to literature methods. Macrolactone precursors were prepared according to the following scheme:
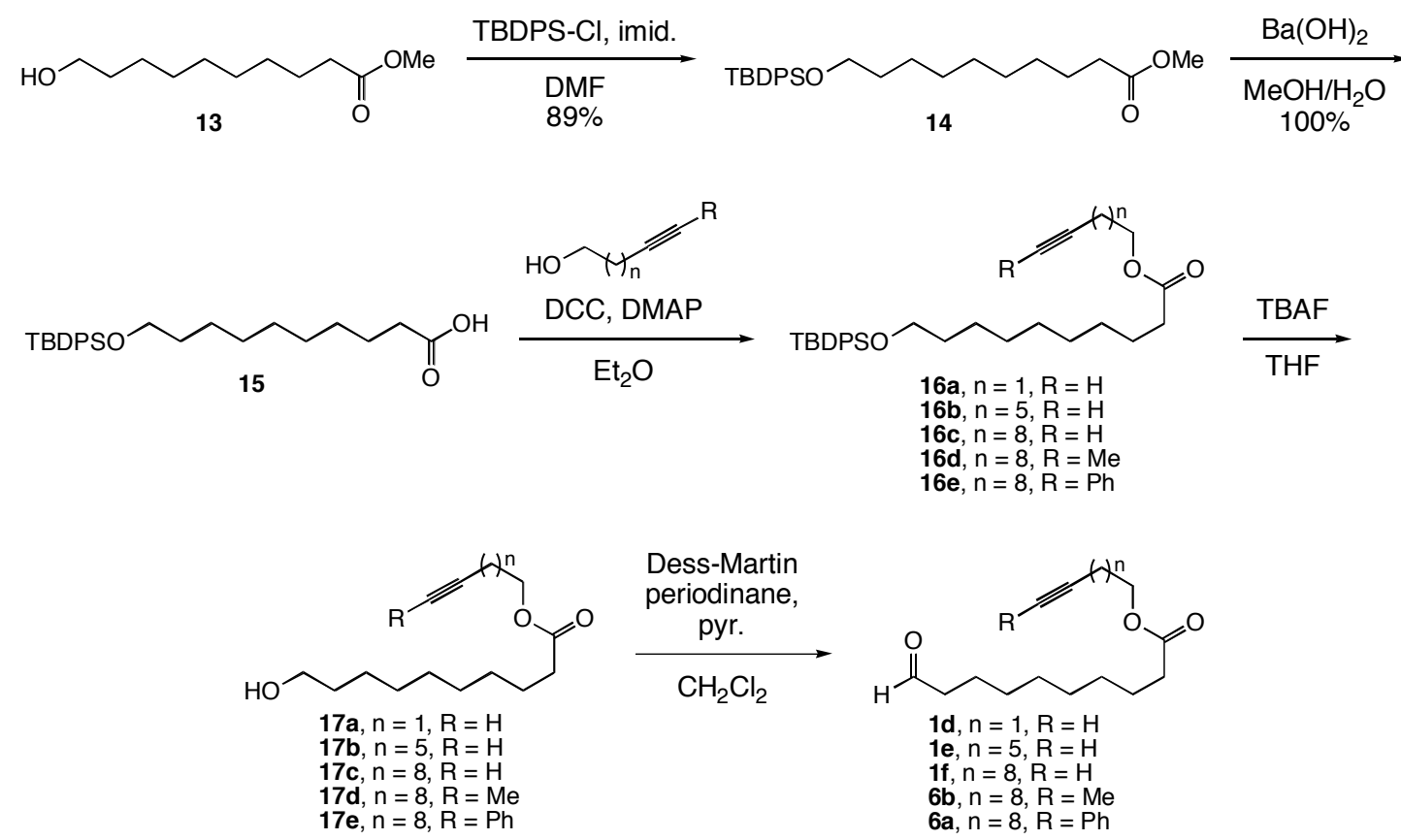

\section{0(tert-Butyl-diphenyl-silanyloxy)-decanoic Acid Methyl Ester (14).}

TBDPSO OMe To a solution of alcohol $13(1.00 \mathrm{~g}, 4.95 \mathrm{mmol})$ in DMF (36 mL) was added TBDPS-Cl (1.63 g, $5.94 \mathrm{mmol})$ followed by imidazole $(0.808 \mathrm{~g}, 11.9 \mathrm{mmol})$. The resulting solution was then stirred at $\mathrm{rt}$ for $2 \mathrm{hr}$. The reaction mixture was diluted with ether $(100 \mathrm{~mL})$ and washed with brine (3 x $100 \mathrm{~mL}$ ). The ethereal layer was dried over $\mathrm{MgSO}_{4}$, filtered and concentrated in vacuo. The crude product was purified by column chromatography $\left(5 \% \mathrm{Et}_{2} \mathrm{O} /\right.$ hexanes $)$ to give the desired TBDPS-ether as a light colored oil (1.89 g, 89\%). ${ }^{1} \mathrm{H}$ NMR $\left(\mathrm{CDCl}_{3}, 400\right.$ $\mathrm{MHz}) \delta$ 7.68-7.65 (m, 4H), 7.41-7.35 (m, 6H), 3.67 (s, 3H), $3.65(\mathrm{t}, J=6.5 \mathrm{~Hz}, 2 \mathrm{H}), 2.30$ $(\mathrm{t}, J=7.3 \mathrm{~Hz}, 2 \mathrm{H}), 1.64-1.58(\mathrm{~m}, 2 \mathrm{H}), 1.58-1.52(\mathrm{~m}, 2 \mathrm{H}), 1.36-1.23(\mathrm{~m}, 10 \mathrm{H}), 1.05$ (s, $9 \mathrm{H}) ;{ }^{13} \mathrm{C} \mathrm{NMR}\left(\mathrm{CDCl}_{3}, 100 \mathrm{MHz}\right) \delta 174.3,135.6,134.1,129.4,127.5,63.9,51.4,34.1$, $32.5,29.4,29.3,29.2,29.1,26.9,25.7,24.9,19.2$; IR (thin film) $v 2929,2855,1740$, 
1471, 1427, 1170, 1110, $701 \mathrm{~cm}^{-1}$. HRMS (ES+) calcd. for $\mathrm{C}_{27} \mathrm{H}_{40} \mathrm{O}_{3} \mathrm{SiNa}[\mathrm{M}+\mathrm{Na}]^{+}$ 463.2644, found: 463.2642 .

\section{0-(tert-Butyl-diphenyl-silanyloxy)-decanoic Acid (15).}

TBDPSO
OH $\begin{aligned} & \text { To a solution of methyl ester } 14(1.89 \mathrm{~g}, 4.28 \mathrm{mmol}) \text { in } \\ & 10: 1 \mathrm{MeOH} / \mathrm{H}_{2} \mathrm{O}(40 \mathrm{~mL}) \text { was added } \mathrm{Ba}(\mathrm{OH})_{2}(3.66 \mathrm{~g} \text {, }\end{aligned}$ $21.4 \mathrm{mmol}$ ). The reaction mixture was then stirred at room temperature for $2 \mathrm{~h}$. The reaction mixture was quenched with $1 \mathrm{~N} \mathrm{HCl}(50 \mathrm{~mL})$ and then extracted with ethyl acetate $(5 \times 50 \mathrm{~mL})$. Combined organic extracts were then dried over $\mathrm{MgSO}_{4}$, filtered, and concentrated to give the pure acid $(1.82 \mathrm{~g}, 100 \%)$. ${ }^{1} \mathrm{H} \mathrm{NMR}\left(\mathrm{CDCl}_{3}, 400 \mathrm{MHz}\right) \delta$ 7.70-7.67 (m, 4H), 7.43-7.37 (m, 6H), $3.67(\mathrm{t}, J=6.5 \mathrm{~Hz}, 2 \mathrm{H}), 2.36(\mathrm{t}, J=7.3 \mathrm{~Hz}, 2 \mathrm{H})$, 1.64 (pent, $J=6.5 \mathrm{~Hz}, 2 \mathrm{H}), 1.38-1.25(\mathrm{~m}, 10 \mathrm{H}), 1.07$ (s, 9H); ${ }^{13} \mathrm{C} \mathrm{NMR}\left(\mathrm{CDCl}_{3}, 100\right.$ MHz) $\delta 180.1,135.6,134.2,129.5,127.5,64.0,34.1,32.5,29.4,29.3,29.2,29.0,26.9$, 25.7, 24.7, 19.2; IR (thin film) v 3070, 3048, 2929, 2856, 1708, 1428, 1111, 823, 739, 701, $612 \mathrm{~cm}^{-1}$. HRMS (ES+) calcd. for $\mathrm{C}_{26} \mathrm{H}_{38} \mathrm{O}_{3} \mathrm{SiNa}[\mathrm{M}+\mathrm{Na}]^{+} 449.2488$, found: 449.2499 .

\section{0-(tert-Butyl-diphenyl-silanyloxy)-decanoic Acid But-3-ynyl Ester (16a).}

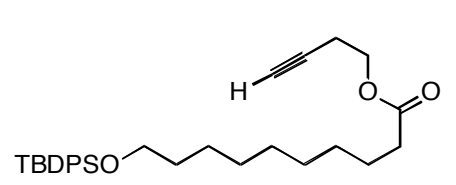

To a solution of acid $15(0.415 \mathrm{~g}, 0.974 \mathrm{mmol})$ in ether $(6$ $\mathrm{mL})$, 3-butyn-1-ol (0.075 g, $1.1 \mathrm{mmol})$, DCC (0.220 g, 1.07 mmol), and DMAP (0.012 g, $0.097 \mathrm{mmol})$ were added. The resulting solution was then stirred for $16 \mathrm{~h}$ at $\mathrm{rt}$. The reaction mixture was then filtered and concentrated in vacuo. The crude residue was then purified via column chromatography (5\% ether/hexanes) to give the desired ester 16a as a light colored oil (0.419 g, 90\%). ${ }^{1} \mathrm{H}$ NMR $\left(\mathrm{CDCl}_{3}, 400 \mathrm{MHz}\right) \delta$ 7.69-7.65 (m, 4H), 7.44-7.36 (m, 6H), $4.18(\mathrm{t}, J=6.5 \mathrm{~Hz}, 2 \mathrm{H}), 3.65(\mathrm{t}, J=6.5 \mathrm{~Hz}, 2 \mathrm{H}), 2.53(\mathrm{ddd}, J=7.3,6.52 .4 \mathrm{~Hz}, 2 \mathrm{H}), 2.32$ (t, $J=7.3 \mathrm{~Hz}, 2 \mathrm{H}), 1.99$ (t, $J=2.4 \mathrm{~Hz}, 1 \mathrm{H}), 1.65-1.53(\mathrm{~m}, 4 \mathrm{H}), 1.38-1.26(\mathrm{~m}, 10 \mathrm{H}), 1.06$ (s, 9H); ${ }^{13} \mathrm{C} \mathrm{NMR}\left(\mathrm{CDCl}_{3}, 100 \mathrm{MHz}\right) \delta$ 173.6, 135.6, 134.2, 129.5, 127.6, 80.1, 69.8, $64.0,61.9,34.2,32.6,29.4,29.3,29.2,29.1,26.9,25.7,24.9,19.2,19.0$; IR (thin film) $v$ 2929, 2855, 1739, 1471, 1427, 1388, 1237, 1167, 1110, 825, 739, $702 \mathrm{~cm}^{-1}$. HRMS (ES+) calcd. for $\mathrm{C}_{30} \mathrm{H}_{42} \mathrm{O}_{3} \mathrm{SiNa}[\mathrm{M}+\mathrm{Na}]^{+}$501.2801, found: 501.2819. 


\section{0-(tert-Butyl-diphenyl-silanyloxy)-decanoic Acid Oct-7-ynyl Ester (16b).}

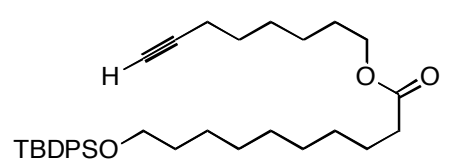

To a solution of acid $15(0.418 \mathrm{~g}, 0.981 \mathrm{mmol})$ in ether $(6$

mL), 7-octyn-1-ol (0.135 g, $1.08 \mathrm{mmol})$, DCC (0.222 g, 1.08 mmol), and DMAP (0.012 g, $0.098 \mathrm{mmol})$ were added. The

resulting solution was then stirred for $16 \mathrm{~h}$ at $\mathrm{rt}$. The reaction mixture was then filtered and concentrated in vacuo. The crude residue was then purified via column chromatography (5\% ether/hexanes) to give the desired ester $\mathbf{1 6 b}$ as a light colored oil (0.436 g, 93\%). ${ }^{1} \mathrm{H}$ NMR $\left(\mathrm{CDCl}_{3}, 400 \mathrm{MHz}\right) \delta$ 7.69-7.67 (m, 4H), 7.44-7.36 (m, 6H), $4.08(\mathrm{t}, J=6.5 \mathrm{~Hz}, 2 \mathrm{H}), 3.66(\mathrm{t}, J=7.3 \mathrm{~Hz}, 2 \mathrm{H}), 2.30(\mathrm{t}, J=7.3 \mathrm{~Hz}, 2 \mathrm{H}), 2.20$ (ddd, $J=$ 7.3, 6.5, $2.4 \mathrm{~Hz}, 2 \mathrm{H}), 1.94(\mathrm{t}, J=2.4 \mathrm{~Hz}, 1 \mathrm{H}), 1.68-1.51(\mathrm{~m}, 8 \mathrm{H}), 1.48-1.26(\mathrm{~m}, 14 \mathrm{H})$, $1.06(\mathrm{~s}, 9 \mathrm{H}) ;{ }^{13} \mathrm{C} \mathrm{NMR}\left(\mathrm{CDCl}_{3}, 100 \mathrm{MHz}\right) \delta 173.9,135.5,134.1,129.4,127.5,84.4,68.3$, 64.2, 63.9, 34.4, 32.5, 29.4, 29.3, 29.2, 29.1, 28.5, 28.3, 26.8, 25.7, 25.4, 25.0, 19.2, 18.3; IR (thin film) v 3309, 2930, 2856, 1735, 1463, 1427, 1388, 1360, 1171, 1110, 823, 702, $613 \mathrm{~cm}^{-1}$. HRMS (ES+) calcd. for $\mathrm{C}_{34} \mathrm{H}_{50} \mathrm{O}_{3} \mathrm{SiNa}[\mathrm{M}+\mathrm{Na}]^{+}$557.3247, found: 557.3422.

\section{0-(tert-Butyl-diphenyl-silanyloxy)-decanoic Acid Dodec-10-ynyl Ester (16c).}

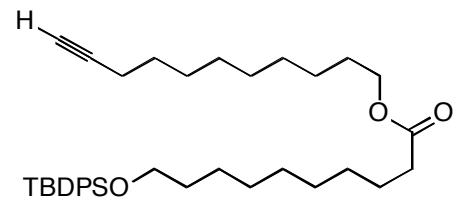

To a solution of acid 15 (0.181 g, $0.424 \mathrm{mmol})$ in ether (2 $\mathrm{mL}), 10$-undecyn-1-ol (0.078 g, $0.47 \mathrm{mmol})$, DCC (0.096 g, $0.47 \mathrm{mmol})$, and DMAP (0.005 g, $0.04 \mathrm{mmol})$ were added. The resulting solution was then stirred for $16 \mathrm{~h}$ at $\mathrm{rt}$. The reaction mixture was then filtered and concentrated in vacuo. The crude residue was then purified via column chromatography (5\% ether/hexanes) to give the desired ester 16c as a light colored oil (0.214 g, 88\%). ${ }^{1} \mathrm{H} \mathrm{NMR}\left(\mathrm{CDCl}_{3}, 400 \mathrm{MHz}\right) \delta$ 7.68-7.66 (m, 4H), 7.42-7.35 (m, 6H), $4.06(\mathrm{t}, J=6,5 \mathrm{~Hz}, 2 \mathrm{H}), 3.66(\mathrm{t}, J=6.5 \mathrm{~Hz}, 2 \mathrm{H}), 2.29(\mathrm{t}, J=7.3 \mathrm{~Hz}, 2 \mathrm{H}), 2.18(\mathrm{ddd}, J=$ 7.3, 6.5, 2.4 Hz, 2H), $1.93(\mathrm{t}, J=2.4 \mathrm{~Hz}, 1 \mathrm{H}), 1.64-1.49(\mathrm{~m}, 8 \mathrm{H}), 1.44-1.27(\mathrm{~m}, 20 \mathrm{H})$, 1.06 (s, 9H); ${ }^{13} \mathrm{C} \mathrm{NMR}\left(\mathrm{CDCl}_{3}, 100 \mathrm{MHz}\right) \delta 174.0,135.6,134.2$, 129.4, 127.5, 84.7, 68.1, 64.33, 64.0, 34.4, 32.6, 29.4, 29.3, 29.2, 29.1, 29.0, 28.7, 28.6, 28.4, 26.9, 25.9, 25.7, 25.0, 19.2, 18.4; IR (thin film) v 2929, 2855, 1735, 1463, 1427, 1389, 1360, 1173, 1110 $\mathrm{cm}^{-1}$. HRMS (ES+) calcd. for $\mathrm{C}_{37} \mathrm{H}_{56} \mathrm{O}_{3} \mathrm{SiNa}[\mathrm{M}+\mathrm{Na}]^{+}, 599.3896$ found: 599.3891 . 


\section{0-(tert-Butyl-diphenyl-silanyloxy)-decanoic Acid Dodec-10-ynyl Ester (16d).}

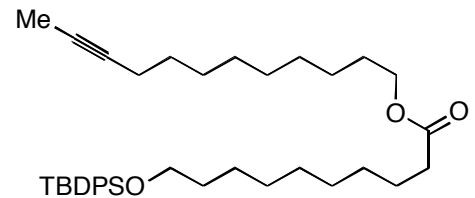

To a solution of acid $15(0.809 \mathrm{~g}, 1.90 \mathrm{mmol})$ in ether (9 $\mathrm{mL})$, dodec-10-yn-1-ol (0.315 g, $1.73 \mathrm{mmol})$, DCC (0.391 $\mathrm{g}, 1.90 \mathrm{mmol})$, and DMAP $(0.022 \mathrm{~g}, 0.17 \mathrm{mmol})$ were added. The resulting solution was then stirred for $16 \mathrm{~h}$ at $\mathrm{rt}$. The reaction mixture was then filtered and concentrated in vacuo. The crude residue was then purified via column chromatography (5\% ether/hexanes) to give the desired ester $16 \mathbf{d}$ as a light colored oil (0.999 g, 98\%). ${ }^{1} \mathrm{H} \mathrm{NMR}\left(\mathrm{CDCl}_{3}, 400 \mathrm{MHz}\right) \delta$ 7.68-7.66 (m, 4H), 7.44-7.35 (m, 6H), $4.06(\mathrm{t}, J=6.5 \mathrm{~Hz}, 2 \mathrm{H}), 3.65(\mathrm{t}, J=6.5 \mathrm{~Hz}, 2 \mathrm{H}), 2.29(\mathrm{t}, J=7.3 \mathrm{~Hz}, 2 \mathrm{H}), 2.14-2.08(\mathrm{~m}$, 2H), $1.78(\mathrm{t}, J=2.4 \mathrm{~Hz}, 3 \mathrm{H}), 1.65-1.59(\mathrm{~m}, 4 \mathrm{H}), 1.57-1.52(\mathrm{~m}, 2 \mathrm{H}), 1.47$ (pent, $J=7.3$ $\mathrm{Hz}, 2 \mathrm{H}), 1.38-1.24(\mathrm{~m}, 20 \mathrm{H}), 1.05(\mathrm{~s}, 9 \mathrm{H}) ;{ }^{13} \mathrm{C} \mathrm{NMR}\left(\mathrm{CDCl}_{3}, 100 \mathrm{MHz}\right) \delta 174.0,135.5$, 134.1, 129.4, 127.5, 79.3, 75.3, 64.4, 64.0, 34.4, 32.5, 29.4, 29.3, 29.2, 29.1, 29.0, 28.8, 28.6, 26.8, 25.9, 25.7, 25.0, 19.2, 18.7, 3.5; IR (thin film) v 2929, 2855, 1736, 1463, 1427, 1172, 1110, 739, 702, $687 \mathrm{~cm}^{-1}$. HRMS (ES+) calcd. for $\mathrm{C}_{38} \mathrm{H}_{58} \mathrm{O}_{3} \mathrm{SiNa}[\mathrm{M}+\mathrm{Na}]^{+}$ 613.4053, found: 613.4051 .

\section{0-(tert-Butyl-diphenyl-silanyloxy)-decanoic Acid 11-Phenyl-Undec-10-ynyl Ester}

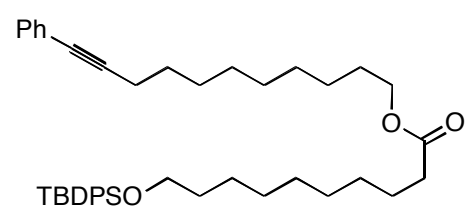
(16e).

To a solution of acid 15 (0.600 g, $1.41 \mathrm{mmol})$ in ether (8 mL), 11-phenyl-undec-10-yn-1-ol (0.375 g, $1.54 \mathrm{mmol})$,

$\operatorname{DCC}(0.319 \mathrm{~g}, 1.54 \mathrm{mmol})$, and DMAP $(0.018 \mathrm{~g}, 0.14 \mathrm{mmol})$ were added. The resulting solution was then stirred for $16 \mathrm{~h}$ at $\mathrm{rt}$. The reaction mixture was then filtered and concentrated in vacuo. The crude residue was then purified via column chromatography (5\% ether/hexanes) to give the desired ester 16e as a light colored oil $(0.758 \mathrm{~g}, 82 \%) .{ }^{1} \mathrm{H}$ NMR $\left(\mathrm{CDCl}_{3}, 500 \mathrm{MHz}\right) \delta$ 7.72-7.70 (m, 4H), 7.46-7.39 (m, 8H), 7.31-7.26 (m, 3H), $4.10(\mathrm{t}, J=7.1 \mathrm{~Hz}, 2 \mathrm{H}), 3.69(\mathrm{t}, J=6.6 \mathrm{~Hz}, 2 \mathrm{H}), 2.43(\mathrm{t}, J=7.1 \mathrm{~Hz}, 2 \mathrm{H}), 2.32(\mathrm{t}, J=7.1$ $\mathrm{Hz}, 2 \mathrm{H}), 1.68-1.56(\mathrm{~m}, 8 \mathrm{H}), 1.50-1.46(\mathrm{~m}, 2 \mathrm{H}), 1.40-1.36(\mathrm{~m}, 11 \mathrm{H}), 1.32-1.29(\mathrm{~m}, 7 \mathrm{H})$, 1.09 (s, 9H); ${ }^{13} \mathrm{C} \mathrm{NMR}\left(\mathrm{CDCl}_{3}, 125 \mathrm{MHz}\right) \delta 173.9,135.5,134.1,131.5,129.4,128.1$, 127.5, 127.4, 127.0, 90.3, 80.6, 64.3, 63.9, 34.3, 32.5, 29.4, 29.3, 29.2, 29.1, 29.0, 28.8, 28.7, 28.6, 26.8, 25.9, 25.7, 25.0, 19.3, 19.2; IR (thin film) v 2929, 2855, 1735, 1489, 
1463, 1427, 1238, 1174, 1111, 756, 740, $702 \mathrm{~cm}^{-1}$. HRMS (ES+) calcd. for $\mathrm{C}_{43} \mathrm{H}_{60} \mathrm{O}_{3} \mathrm{SiNa}[\mathrm{M}+\mathrm{Na}]^{+}$675.4205, found: 675.4208 .

\section{0-Hydroxy-decanoic Acid But-3-ynyl Ester (17a).}

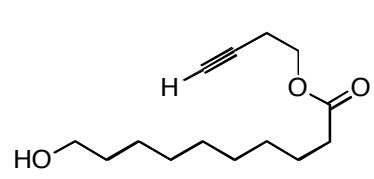

To a solution of TBDPS-ether $16 \mathbf{a}(0.317 \mathrm{~g}, 0.663 \mathrm{mmol})$ in THF

(4 mL) was added TBAF (0.99 $\mathrm{mL}$ of a $1 \mathrm{M}$ solution in THF, 0.99 mmol). The resulting solution was then stirred at $\mathrm{rt}$ for $16 \mathrm{~h}$. The reaction mixture was diluted with ether $(10 \mathrm{~mL})$ and washed with $\mathrm{NaHCO}_{3}(10 \mathrm{~mL})$. The ethereal layer was then dried over $\mathrm{MgSO}_{4}$, filtered and concentrated. The crude product was then purified via column chromatography (30\% ethyl acetate/hexanes) to give 0.158 g (99\%). ${ }^{1} \mathrm{H} \mathrm{NMR}\left(\mathrm{CDCl}_{3}, 400 \mathrm{MHz}\right) \delta 4.16(\mathrm{t}, J=6.5 \mathrm{~Hz}, 2 \mathrm{H}), 3.60(\mathrm{t}, J=7.3 \mathrm{~Hz}, 2 \mathrm{H})$, $2.51(\mathrm{ddd}, J=7.3,6.5,2.4 \mathrm{~Hz}, 2 \mathrm{H}), 2.30(\mathrm{t}, J=7.3 \mathrm{~Hz}, 2 \mathrm{H}), 1.98(\mathrm{t}, J=2.4 \mathrm{~Hz}, 1 \mathrm{H})$, 1.62-1.50 (m, 5H), 1.34-1.27 (m, 10H); ${ }^{13} \mathrm{C} \mathrm{NMR}\left(\mathrm{CDCl}_{3}, 100 \mathrm{MHz}\right) \delta$ 173.6, 80.1, 69.8, $62.9,61.9,34.1,32.7,29.3,29.3,29.1,29.0,25.6,24.8,18.9$; IR (thin film) v 3278, 2916, 2850, 1739, 1471, 1463, 1411, 1266, 1181, 1072, 734, 704, $640 \mathrm{~cm}^{-1}$. HRMS (ES+) calcd. for $\mathrm{C}_{14} \mathrm{H}_{24} \mathrm{O}_{3} \mathrm{Na}[\mathrm{M}+\mathrm{Na}]^{+} 263.1623$, found: 263.1625 .

\section{0-Hydroxy-decanoic Acid Oct-7-ynyl Ester (17b).}

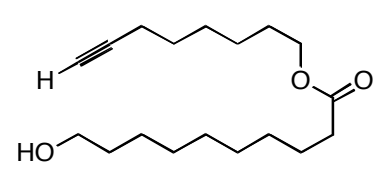

To a solution of TBDPS-ether $\mathbf{1 6 b}(0.736 \mathrm{~g}, 2.14 \mathrm{mmol})$ in THF ( $7 \mathrm{~mL})$ was added TBAF $(2.1 \mathrm{~mL}$ of a $1 \mathrm{M}$ solution in THF, 2.1 $\mathrm{mmol})$. The resulting solution was then stirred at $\mathrm{rt}$ for $16 \mathrm{~h}$. The reaction mixture was diluted with ether $(15 \mathrm{~mL})$ and washed with $\mathrm{NaHCO}_{3}(15 \mathrm{~mL})$. The ethereal layer was then dried over $\mathrm{MgSO}_{4}$, filtered and concentrated. The crude product was then purified via column chromatography (30\% ethyl acetate/hexanes) to give 0.406 $\mathrm{g}(96 \%) .{ }^{1} \mathrm{H} \mathrm{NMR}\left(\mathrm{CDCl}_{3}, 500 \mathrm{MHz}\right) \delta 4.02(\mathrm{t}, J=6.6 \mathrm{~Hz}, 2 \mathrm{H}), 3.56(\mathrm{t}, J=6.6 \mathrm{~Hz}, 2 \mathrm{H})$, $2.23(\mathrm{t}, J=7.6 \mathrm{~Hz}, 2 \mathrm{H}), 2.14(\mathrm{dt}, J=6.6,2.5 \mathrm{~Hz}, 2 \mathrm{H}), 1.89(\mathrm{t}, J=2.5 \mathrm{~Hz}, 1 \mathrm{H}), 1.60-1.46$ $(\mathrm{m}, 8 \mathrm{H}), 1.43-1.35(\mathrm{~m}, 2 \mathrm{H}), 1.34-1.24(\mathrm{~m}, 12 \mathrm{H}) ;{ }^{13} \mathrm{C} \mathrm{NMR}\left(\mathrm{CDCl}_{3}, 125 \mathrm{MHz}\right) \delta 173.9$, $84.3,68.2,64.1,62.7,34.2,32.6,29.3,29.2,29.0,28.4,28.2,25.6,25.3,24.8,18.2$; IR $\left(\right.$ thin film) $v$ 3309, 2930, 2856, 1734, 1464, 1351, 1248, 1177, 1099, 1056, $627 \mathrm{~cm}^{-1}$. HRMS (ES+) calcd. for $\mathrm{C}_{18} \mathrm{H}_{32} \mathrm{O}_{3} \mathrm{Na}[\mathrm{M}+\mathrm{Na}]^{+}$319.2249, found: 319.2245. 


\section{0-Hydroxy-decanoic Acid Undec-10-ynyl Ester (17c).}

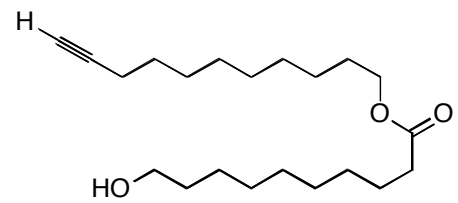

To a solution of TBDPS-ether 16c $(1.03 \mathrm{~g}, 1.78 \mathrm{mmol})$ in THF $(9 \mathrm{~mL})$ was added TBAF $(2.7 \mathrm{~mL}$ of a $1 \mathrm{M}$ solution in THF, $2.7 \mathrm{mmol}$ ). The resulting solution was then stirred at rt for $16 \mathrm{~h}$. The reaction mixture was diluted with ether $(20 \mathrm{~mL})$ and washed with $\mathrm{NaHCO}_{3}(20 \mathrm{~mL})$. The ethereal layer was then dried over $\mathrm{MgSO}_{4}$, filtered and concentrated. The crude product was then purified via column chromatography $(30 \%$ ethyl acetate/hexanes) to give $0.584 \mathrm{~g}(97 \%)$. ${ }^{1} \mathrm{H} \mathrm{NMR}\left(\mathrm{CDCl}_{3}, 500 \mathrm{MHz}\right) \delta 4.04(\mathrm{t}, J=$ $6.6 \mathrm{~Hz}, 2 \mathrm{H}), 3.60$ (t, $J=6.6 \mathrm{~Hz}, 2 \mathrm{H}), 2.26$ (t, $J=7.6 \mathrm{~Hz}, 2 \mathrm{H}$ ), 2.17 (dt, $J=6.6,2.5 \mathrm{~Hz}$, $2 \mathrm{H}), 1.92(\mathrm{t}, J=2.5 \mathrm{~Hz}, 1 \mathrm{H}), 1.63-1.49(\mathrm{~m}, 10 \mathrm{H}), 1.44-1.28(\mathrm{~m}, 18 \mathrm{H}) ;{ }^{13} \mathrm{C} \mathrm{NMR}\left(\mathrm{CDCl}_{3}\right.$, $125 \mathrm{MHz}) \delta$ 173..9, 84.4, 68.2, 64.2, 62.9, 34.3, 32.7, 29.3, 29.1, 29.0, 28.5, 28.3, 25.7, 25.4, 24.9, 18.3; IR (thin film) v 3356, 2928, 2854, 1735, 1465, 1176, $1056 \mathrm{~cm}^{-1}$. HRMS (ES+) calcd. for $\mathrm{C}_{21} \mathrm{H}_{38} \mathrm{O}_{3} \mathrm{Na}[\mathrm{M}+\mathrm{Na}]^{+} 361.2719$, found: 361.2726 .

\section{0-Hydroxy-decanoic Acid Dodec-10-ynyl Ester (17d).}

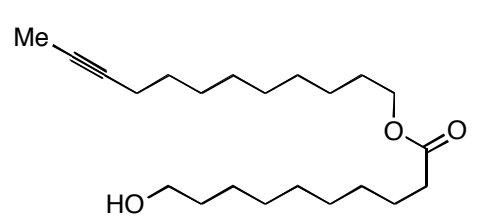

To a solution of TBDPS-ether $16 \mathrm{~d}(0.893 \mathrm{~g}, 1.51 \mathrm{mmol})$ in THF $(8 \mathrm{~mL})$ was added TBAF $(2.3 \mathrm{~mL}$ of a $1 \mathrm{M}$ solution in THF, $2.3 \mathrm{mmol}$ ). The resulting solution was then stirred at $\mathrm{rt}$ for $16 \mathrm{~h}$. The reaction mixture was diluted with ether $(20$ $\mathrm{mL})$ and washed with $\mathrm{NaHCO}_{3}(20 \mathrm{~mL})$. The ethereal layer was then dried over $\mathrm{MgSO}_{4}$, filtered and concentrated. The crude product was then purified via column chromatography (30\% ethyl acetate/hexanes) to give $0.527 \mathrm{~g}(99 \%) .{ }^{1} \mathrm{H} \mathrm{NMR}\left(\mathrm{CDCl}_{3}\right.$, $500 \mathrm{MHz}) \delta 3.96(\mathrm{t}, J=7.1 \mathrm{~Hz}, 2 \mathrm{H}), 3.53(\mathrm{t}, J=7.1 \mathrm{~Hz}, 2 \mathrm{H}), 2.52(\mathrm{bs}, 1 \mathrm{H}), 2.20(\mathrm{t}, J=$ $7.1 \mathrm{~Hz}, 2 \mathrm{H}), 2.05-2.01(\mathrm{~m}, 2 \mathrm{H}), 1.69(\mathrm{t}, J=2.5 \mathrm{~Hz}, 3 \mathrm{H}), 1.56-1.44(\mathrm{~m}, 6 \mathrm{H}), 1.42-1.35$ (m, 2H), 1.30-1.19 (m, 20H); ${ }^{13} \mathrm{C}$ NMR $\left(\mathrm{CDCl}_{3}, 125 \mathrm{MHz}\right) \delta$ 173..9, 84.4, 68.2, 64.2, $62.9,34.3,32.7,29.3,29.1,29.0,28.5,28.3,25.7,25.4,24.9,18.3$; IR (thin film) v 3356, $2928,2854,1735,1465,1176,1056 \mathrm{~cm}^{-1}$. HRMS (ES+) calcd. for $\mathrm{C}_{22} \mathrm{H}_{40} \mathrm{O}_{3} \mathrm{Na}[\mathrm{M}+\mathrm{Na}]^{+}$ 375.2875, found: 375.2874 . 


\section{0-Hydroxy-decanoic Acid 11-Phenyl-undec-10-ynyl Ester (17e).}

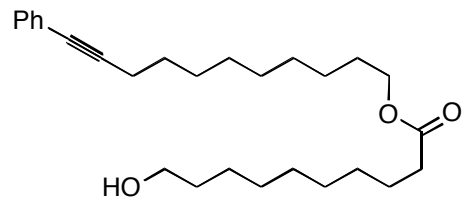

To a solution of TBDPS-ether $16 \mathrm{e}(0.312 \mathrm{~g}, 0.478 \mathrm{mmol})$ in

THF ( $3 \mathrm{~mL})$ was added TBAF $(0.72 \mathrm{~mL}$ of a $1 \mathrm{M}$ solution in THF, $0.72 \mathrm{mmol})$. The resulting solution was then stirred at $\mathrm{rt}$ for $16 \mathrm{~h}$. The reaction mixture was diluted with ether $(10 \mathrm{~mL})$ and washed with $\mathrm{NaHCO}_{3}(10 \mathrm{~mL})$. The ethereal layer was then dried over $\mathrm{MgSO}_{4}$, filtered and concentrated. The crude product was then purified via column chromatography $(30 \%$ ethyl acetate/hexanes) to give $0.183 \mathrm{~g}(93 \%) .{ }^{1} \mathrm{H}$ NMR $\left(\mathrm{CDCl}_{3}, 500 \mathrm{MHz}\right) \delta$ 7.37-7.35 $(\mathrm{m}, 2 \mathrm{H}), 7.26-7.22(\mathrm{~m}, 3 \mathrm{H}), 4.03(\mathrm{t}, J=7.1 \mathrm{~Hz}, 2 \mathrm{H}), 3.58(\mathrm{t}, J=7.1 \mathrm{~Hz}, 2 \mathrm{H}), 2.37(\mathrm{t}, J=$ $7.1 \mathrm{~Hz}, 2 \mathrm{H}), 2.26$ (t, $J=7.1 \mathrm{~Hz}, 2 \mathrm{H}), 1.99$ (s, 1H), 1.61-1.56 (m, 6H), 1.54-1.49 (m, 2H), 1.43-1.40 (m, 2H), 1.33-1.25 (18H); ${ }^{13} \mathrm{C} \mathrm{NMR}\left(\mathrm{CDCl}_{3}, 125 \mathrm{MHz}\right) \delta 173.9,131.4,128.0$, 127.3, 123.9, 90.2, 80.5, 64.2, 62.7, 34.2, 32.6, 29.2, 29.21, 29.1, 29.0, 29.0, 28.9, 28.7, 28.6, 28.5, 25.8, 25.6, 24.8, 19.2; IR (thin film) v 3398, 2927, 2854, 1734, 1489, 1464, $1175,1056,756,691 \mathrm{~cm}^{-1}$. HRMS (ES+) calcd. for $\mathrm{C}_{37} \mathrm{H}_{42} \mathrm{O}_{3} \mathrm{Na}[\mathrm{M}+\mathrm{Na}]^{+} 437.3032$, found: 437.3030 .

\section{0-Oxo-decanoic Acid But-3-ynyl Ester (1d).}

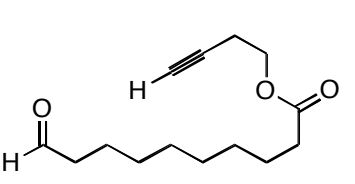

To a solution of alcohol 17a $(0.228 \mathrm{~g}, 0.957 \mathrm{mmol})$ in $4.5 \mathrm{~mL}$ of

$\mathrm{CH}_{2} \mathrm{Cl}_{2}$ and $0.39 \mathrm{~mL}$ of pyridine at $0{ }^{\circ} \mathrm{C}$ was added Dess-Martin periodinane $(1.0 \mathrm{~g}, 2.4 \mathrm{mmol})$. After stirring for $10 \mathrm{~min}, 0.5 \mathrm{~mL}$ of 'wet' $\mathrm{CH}_{2} \mathrm{Cl}_{2}$ (pre-washed with water) was added dropwise. The cooling bath was removed and the mixture stirred at ambient temperature for $1 \mathrm{~h}$. A 1:1 mixture of aqueous $\mathrm{NaHCO}_{3} / \mathrm{Na}_{2} \mathrm{~S}_{2} \mathrm{O}_{3}$ was added and the biphasic mixture stirred vigorously for 20 min. The reaction mixture was then extracted with ether $(3 \times 10 \mathrm{~mL})$. The combined organic extracts were dried over $\mathrm{MgSO}_{4}$, filtered and concentrated. The very sensitive crude aldehyde was then quickly purified via column chromatography (10\% ether/hexanes) to give $0.202 \mathrm{~g}$ of $\mathbf{1 d}(89 \%)$, which was used immediately in the next reaction. ${ }^{1} \mathrm{H} \mathrm{NMR}\left(\mathrm{CDCl}_{3}, 500 \mathrm{MHz}\right) \delta 9.76(\mathrm{t}, J=2.0 \mathrm{~Hz}, 1 \mathrm{H}), 4.18(\mathrm{t}, J=6.5 \mathrm{~Hz}$, 2H), $2.52(\mathrm{dt}, J=6.5,2.5 \mathrm{~Hz}, 2 \mathrm{H}), 2.41(\mathrm{dt}, J=7.6,2.5 \mathrm{~Hz}, 2 \mathrm{H}), 2.32(\mathrm{t}, J=7.6 \mathrm{~Hz}, 2 \mathrm{H})$, $2.00(\mathrm{t}, J=2.5 \mathrm{~Hz}, 1 \mathrm{H}), 1.64-1.59(\mathrm{~m}, 4 \mathrm{H}), 1.34-1.29(\mathrm{~m}, 8 \mathrm{H}) ;{ }^{13} \mathrm{C} \mathrm{NMR}\left(\mathrm{CDCl}_{3}, 125\right.$ MHz) $\delta 202.8,173.5,80.1,69.8,61.9,43.9,34.1,29.1,29.0,28.9,24.8,22.0,19.0$; IR 
(thin film) $\vee 2932,2857,1731,1454,1250,1174,1086 \mathrm{~cm}^{-1}$. MS (ES+) calcd. for $\mathrm{C}_{14} \mathrm{H}_{22} \mathrm{O}_{3} \mathrm{Na}[\mathrm{M}+\mathrm{Na}]^{+}$261.1, found: 261.1.

\section{0-Oxo-decanoic Acid Oct-7-ynyl Ester (1e).}

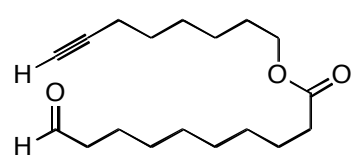

To a solution of alcohol $\mathbf{1 7 b}(0.190 \mathrm{~g}, 0.646 \mathrm{mmol})$ in $3.5 \mathrm{~mL}$ of

$\mathrm{CH}_{2} \mathrm{Cl}_{2}$ and $0.26 \mathrm{~mL}$ of pyridine at $0{ }^{\circ} \mathrm{C}$ was added Dess-Martin periodinane $(0.685 \mathrm{~g}, 1.62 \mathrm{mmol})$. After stirring for $10 \mathrm{~min}, 0.5$

$\mathrm{mL}$ of 'wet' $\mathrm{CH}_{2} \mathrm{Cl}_{2}$ (pre-washed with water) was added dropwise. The cooling bath was removed and the mixture stirred at ambient temperature for $1 \mathrm{~h}$. A 1:1 mixture of aqueous $\mathrm{NaHCO}_{3} / \mathrm{Na}_{2} \mathrm{~S}_{2} \mathrm{O}_{3}$ was added and the biphasic mixture stirred vigorously for 20 min. The reaction mixture was then extracted with ether $(3 \times 10 \mathrm{~mL})$. The combined organic extracts were dried over $\mathrm{MgSO}_{4}$, filtered and concentrated. The very sensitive aldehyde was then quickly purified via column chromatography (10\% ether/hexanes) to give $0.150 \mathrm{~g}$ of $1 \mathrm{e}(80 \%)$, which was used immediately in the next reaction. ${ }^{1} \mathrm{H}$ NMR $\left(\mathrm{CDCl}_{3}, 500 \mathrm{MHz}\right) \delta 9.75(\mathrm{t}, J=1.5 \mathrm{~Hz}, 1 \mathrm{H}), 4.06(\mathrm{t}, J=6.6 \mathrm{~Hz}, 2 \mathrm{H}), 2.41(\mathrm{dt}, J=7.1$, $1.5 \mathrm{~Hz}, 2 \mathrm{H}), 2.28(\mathrm{t}, J=7.6 \mathrm{~Hz}, 2 \mathrm{H}), 2.18(\mathrm{dt}, J=7.1,2.5 \mathrm{~Hz}, 2 \mathrm{H}), 1.93(\mathrm{t}, J=2.5 \mathrm{~Hz}$, $1 \mathrm{H}), 1.66-1.58(\mathrm{~m}, 6 \mathrm{H}), 1.56-1.50(\mathrm{~m}, 2 \mathrm{H}), 1.46-1.40(\mathrm{~m}, 1 \mathrm{H}), 1.39-1.34(\mathrm{~m}, 1 \mathrm{H}), 1.32-$ $1.28(\mathrm{~m}, 10 \mathrm{H}) ;{ }^{13} \mathrm{C} \mathrm{NMR}\left(\mathrm{CDCl}_{3}, 125 \mathrm{MHz}\right) \delta 202.8,173.9,84.5,68.2,64.2,43.9,34.3$, $29.1,29.0,28.5,28.3,25.4,24.9,22.0,18.3$; IR (thin film) $v 2929,2855,1731,1470$, 1359, 1248, 1174, $1112 \mathrm{~cm}^{-1}$. MS (ES+) calcd. for $\mathrm{C}_{17} \mathrm{H}_{30} \mathrm{O}_{4} \mathrm{SiNa}[\mathrm{M}+\mathrm{Na}]^{+} 317.2093$, found: 317.2 .

\section{0-Oxo-decanoic Acid Undec-10-ynyl Ester (1f).}

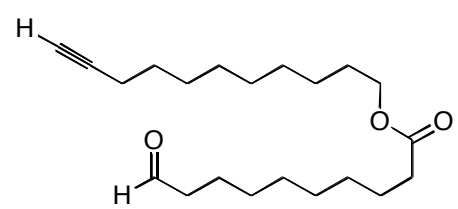

To a solution of alcohol 17c $(0.200 \mathrm{~g}, 0.591 \mathrm{mmol})$ in 2.5 $\mathrm{mL}$ of $\mathrm{CH}_{2} \mathrm{Cl}_{2}$ and $0.24 \mathrm{~mL}$ of pyridine at $0{ }^{\circ} \mathrm{C}$ was added Dess-Martin periodinane $(0.627 \mathrm{~g}, 1.48 \mathrm{mmol})$. After stirring for $10 \mathrm{~min}, 0.5 \mathrm{~mL}$ of 'wet' $\mathrm{CH}_{2} \mathrm{Cl}_{2}$ (pre-washed with water) was added dropwise. The cooling bath was removed and the mixture stirred at ambient temperature for $1 \mathrm{~h}$. A 1:1 mixture of aqueous $\mathrm{NaHCO}_{3} / \mathrm{Na}_{2} \mathrm{~S}_{2} \mathrm{O}_{3}$ was added and the biphasic mixture stirred vigorously for $20 \mathrm{~min}$. The reaction mixture was then extracted with ether $(3 \mathrm{x} 10$ $\mathrm{mL}$ ). The combined organic extracts were dried over $\mathrm{MgSO}_{4}$, filtered and concentrated. 
The very sensitive aldehyde was then quickly purified via column chromatography (10\% ether/hexanes) to give $0.178 \mathrm{~g}$ of $\mathbf{1 f}(90 \%)$, which was used immediately in the next reaction. ${ }^{1} \mathrm{H} \mathrm{NMR}\left(\mathrm{CDCl}_{3}, 400 \mathrm{MHz}\right) \delta 9.75(\mathrm{t}, J=1.6 \mathrm{~Hz}, 1 \mathrm{H}), 4.05(\mathrm{t}, J=6.5 \mathrm{~Hz}$, 2H), $2.41(\mathrm{dt}, J=7.3,1.6 \mathrm{~Hz}, 2 \mathrm{H}), 2.28(\mathrm{t}, J=7.3 \mathrm{~Hz}, 2 \mathrm{H}), 2.18(\mathrm{dt}, J=7.3,2.4 \mathrm{~Hz}, 2 \mathrm{H})$, $1.93(\mathrm{t}, J=2.4 \mathrm{~Hz}, 1 \mathrm{H}), 1.63-1.59(\mathrm{~m}, 6 \mathrm{H}), 1.55-1.48(\mathrm{~m}, 2 \mathrm{H}), 1.39-1.30(\mathrm{~m}, 18 \mathrm{H}) ;{ }^{13} \mathrm{C}$ NMR $\left(\mathrm{CDCl}_{3}, 125 \mathrm{MHz}\right) \delta 202.8,173.9,94.7,84.7,68.1,64.4,43.9,34.3,29.3,29.2$, 29.1, 29.0, 28.7, 28.6, 28.4, 25.9, 24.9, 22.0, 18.4; IR (thin film) $v 2929,2855,1731$, 1464, 1359. 1243, 1174, $1107 \mathrm{~cm}-1$. MS (ES+) calcd. for $\mathrm{C}_{21} \mathrm{H}_{36} \mathrm{O}_{3} \mathrm{Na}[\mathrm{M}+\mathrm{Na}]+359.2$, found: 359.2 .

\section{0-Oxo-decanoic Acid 11-Phenyl-undec-10-ynyl Ester (6a).}

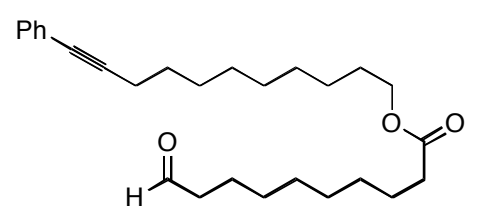

To a solution of alcohol $17 \mathrm{e}(0.200 \mathrm{~g}, 0.484 \mathrm{mmol})$ in 2.5 $\mathrm{mL}$ of $\mathrm{CH}_{2} \mathrm{Cl}_{2}$ and $0.197 \mathrm{~mL}$ of pyridine at $0{ }^{\circ} \mathrm{C}$ was added Dess-Martin periodinane $(0.513 \mathrm{~g}, 1.21 \mathrm{mmol})$. After stirring for $10 \mathrm{~min}, 0.5 \mathrm{~mL}$ of 'wet' $\mathrm{CH}_{2} \mathrm{Cl}_{2}$ (pre-washed with water) was added dropwise. The cooling bath was removed and the mixture stirred at ambient temperature for $1 \mathrm{~h}$. A 1:1 mixture of aqueous $\mathrm{NaHCO}_{3} / \mathrm{Na}_{2} \mathrm{~S}_{2} \mathrm{O}_{3}$ was added and the biphasic mixture stirred vigorously for $20 \mathrm{~min}$. The reaction mixture was then extracted with ether $(3 \times 10$ $\mathrm{mL}$ ). The combined organic extracts were dried over $\mathrm{MgSO}_{4}$, filtered and concentrated. The very sensitive aldehyde was then purified via column chromatography (10\% ether/hexanes) to give $0.178 \mathrm{~g}$ of $\mathbf{6 a}(90 \%)$, which was then used immediately in the next reaction. ${ }^{1} \mathrm{H} \mathrm{NMR}\left(\mathrm{CDCl}_{3}, 500 \mathrm{MHz}\right) \delta 9.75(\mathrm{t}, J=1.5 \mathrm{~Hz}, 1 \mathrm{H}), 7.39-7.37(\mathrm{~m}, 2 \mathrm{H})$, 7.29-7.24 (m, 3H), $4.05(\mathrm{t}, J=6.6 \mathrm{~Hz}, 2 \mathrm{H}), 2.42-2.88(\mathrm{~m}, 4 \mathrm{H}), 2.28(\mathrm{t}, J=7.6 \mathrm{~Hz}, 2 \mathrm{H})$, 1.62-1.58 (m, 8H), 1.46-1.43 (m, 2H), 1.35-1.29 (m, 16H); ${ }^{13} \mathrm{C} \mathrm{NMR}\left(\mathrm{CDCl}_{3}, 125 \mathrm{MHz}\right)$ ठ 202.8, 173.9, 131.5, 128.1, 127.4, 124.1, 90.4, 80.6, 64.4, 43.9, 34.3, 29.4, 29.4, 29.2, 29.1, 29.0, 28.9, 28.7, 28.6, 25.9, 24.9, 22.0, 19.4; IR (thin film) v 2929, 2855, 1731, $1489,1464,1389,1353,1243,1173,1100,1069,756,692 \mathrm{~cm}^{-1}$. MS (ES+) calcd. for $\mathrm{C}_{27} \mathrm{H}_{40} \mathrm{O}_{3} \mathrm{Na}[\mathrm{M}+\mathrm{Na}]^{+}$435.3, found: 435.3. 


\section{0-Oxo-decanoic Acid Dodec-10-ynyl Ester (6b).}

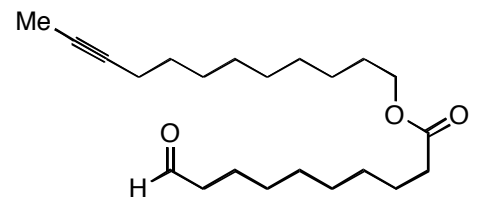

To a solution of alcohol $\mathbf{1 7 d}(0.182 \mathrm{~g}, 0.517 \mathrm{mmol})$ in 2.5 $\mathrm{mL}$ of $\mathrm{CH}_{2} \mathrm{Cl}_{2}$ and $0.210 \mathrm{~mL}$ of pyridine at $0{ }^{\circ} \mathrm{C}$ was added Dess-Martin periodinane $(0.548 \mathrm{~g}, 1.29 \mathrm{mmol})$. After stirring for $10 \mathrm{~min}, 0.5 \mathrm{~mL}$ of 'wet' $\mathrm{CH}_{2} \mathrm{Cl}_{2}$ (pre-washed with water) was added dropwise. The cooling bath was removed and the mixture stirred at ambient temperature for $1 \mathrm{~h}$. A 1:1 mixture of aqueous $\mathrm{NaHCO}_{3} / \mathrm{Na}_{2} \mathrm{~S}_{2} \mathrm{O}_{3}$ was added and the biphasic mixture stirred vigorously for $20 \mathrm{~min}$. The reaction mixture was then extracted with ether ( 3 x 10 $\mathrm{mL}$ ). The combined organic extracts were dried over $\mathrm{MgSO}_{4}$, filtered and concentrated. The crude product was purified via column chromatography (10\% ether/hexanes) to give $0.178 \mathrm{~g}$ of aldehyde $\mathbf{6 b}(901 \%)$. ${ }^{1} \mathrm{H} \mathrm{NMR}\left(\mathrm{CDCl}_{3}, 500 \mathrm{MHz}\right) \delta 9.76(\mathrm{t}, J=2.0 \mathrm{~Hz}, 1 \mathrm{H})$, $4.05(\mathrm{t}, J=7.1 \mathrm{~Hz}, 2 \mathrm{H}), 2.41(\mathrm{dt}, J=7.1 \mathrm{~Hz}, 2 \mathrm{H}), 2.28(\mathrm{t}, J=7.6 \mathrm{~Hz}, 2 \mathrm{H}), 2.13-2.09(\mathrm{~m}$, $2 \mathrm{H}), 1.77(\mathrm{t}, J=2.5 \mathrm{~Hz}, 3 \mathrm{H}), 1.63-1.57(\mathrm{~m}, 6 \mathrm{H}), 1.49-1.43(\mathrm{~m}, 2 \mathrm{H}), 1.38-1.26(\mathrm{~m}, 18 \mathrm{H})$; ${ }^{13} \mathrm{C} \mathrm{NMR}\left(\mathrm{CDCl}_{3}, 125 \mathrm{MHz}\right) \delta 203.6,174.2,79.6,75.6,64.6,44.1,34.6,29.6,29.4,29.3$, 29.1, 28.9, 26.1, 25.2, 22.3, 18.9, 3.7; IR (thin film) v 2928, 2855, 1732, 1590, 1463, 1391, 1172, $1098 \mathrm{~cm}^{-1}$. MS (ES+) calcd. for $\mathrm{C}_{22} \mathrm{H}_{38} \mathrm{O}_{3} \mathrm{Na}[\mathrm{M}+\mathrm{Na}]^{+}$373.2, found: 373.2 .

\section{References}

1) Pangborn, A. B. G., M. A.; Grubbs, R. H.; Rosen, R. K.; Timmers, F. J.

Organometallics 1996, 15, 1518.

2) Colby, E. A. O. B., K. C.; Jamison, T. F. J. Am. Chem. Soc. 2004, 126, 998.

3) Hopf, H. K., A. Chem. Eur. J. 2001, 7, 4378.

4) Oppolzer, W. R., R. N.; El-Sayed, E. J. Org. Chem. 2001, 66, 4766. 


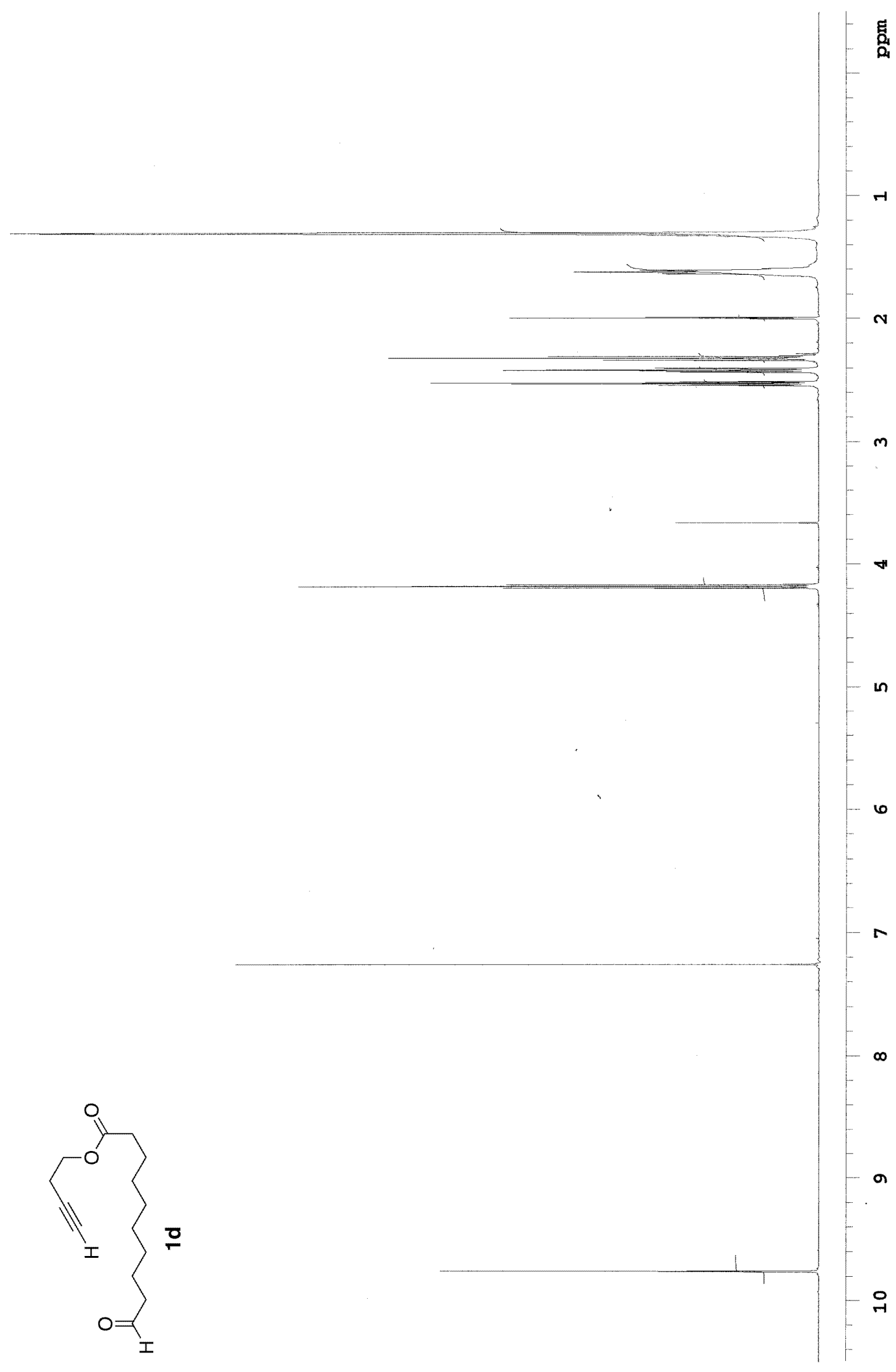




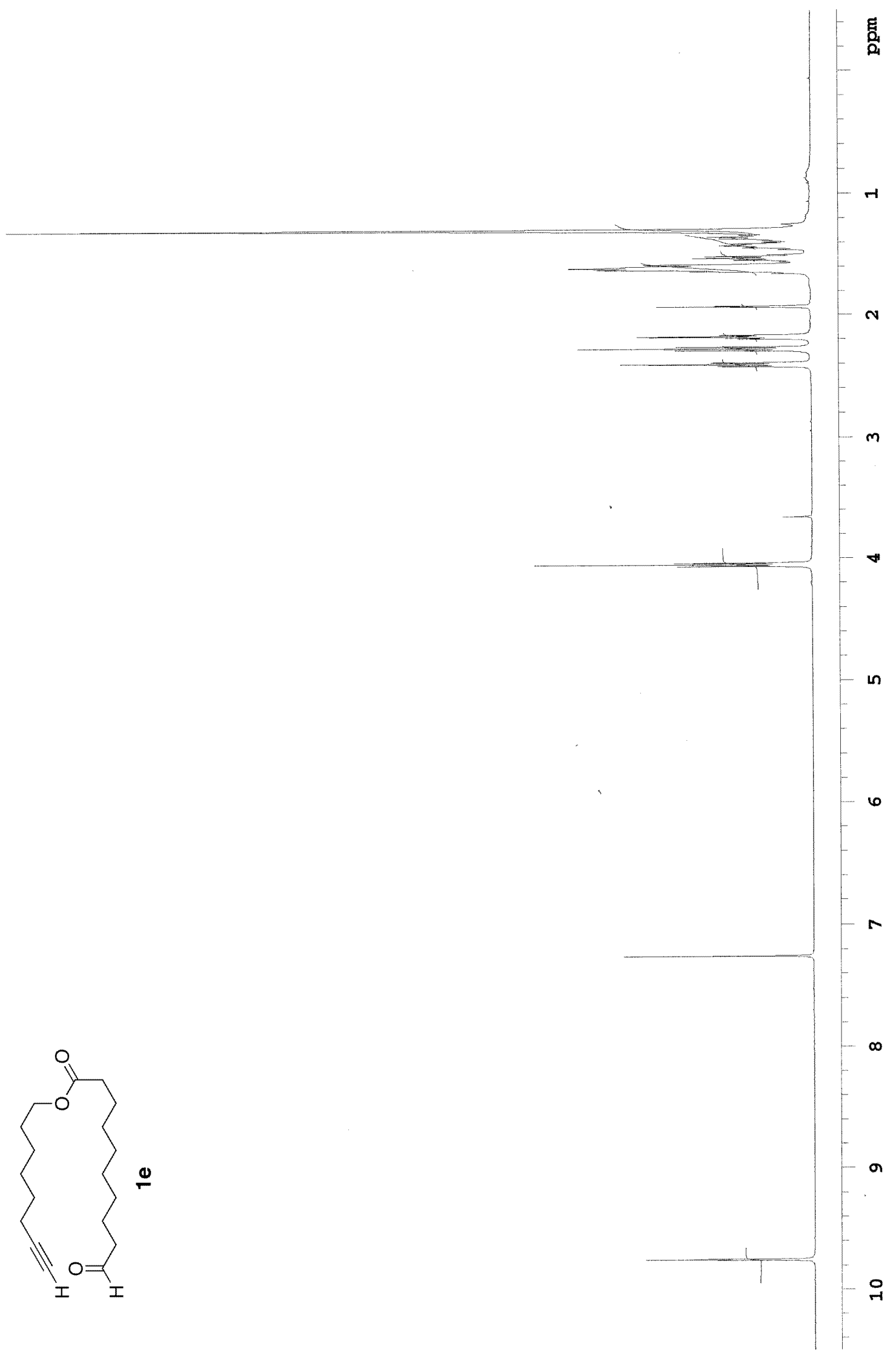




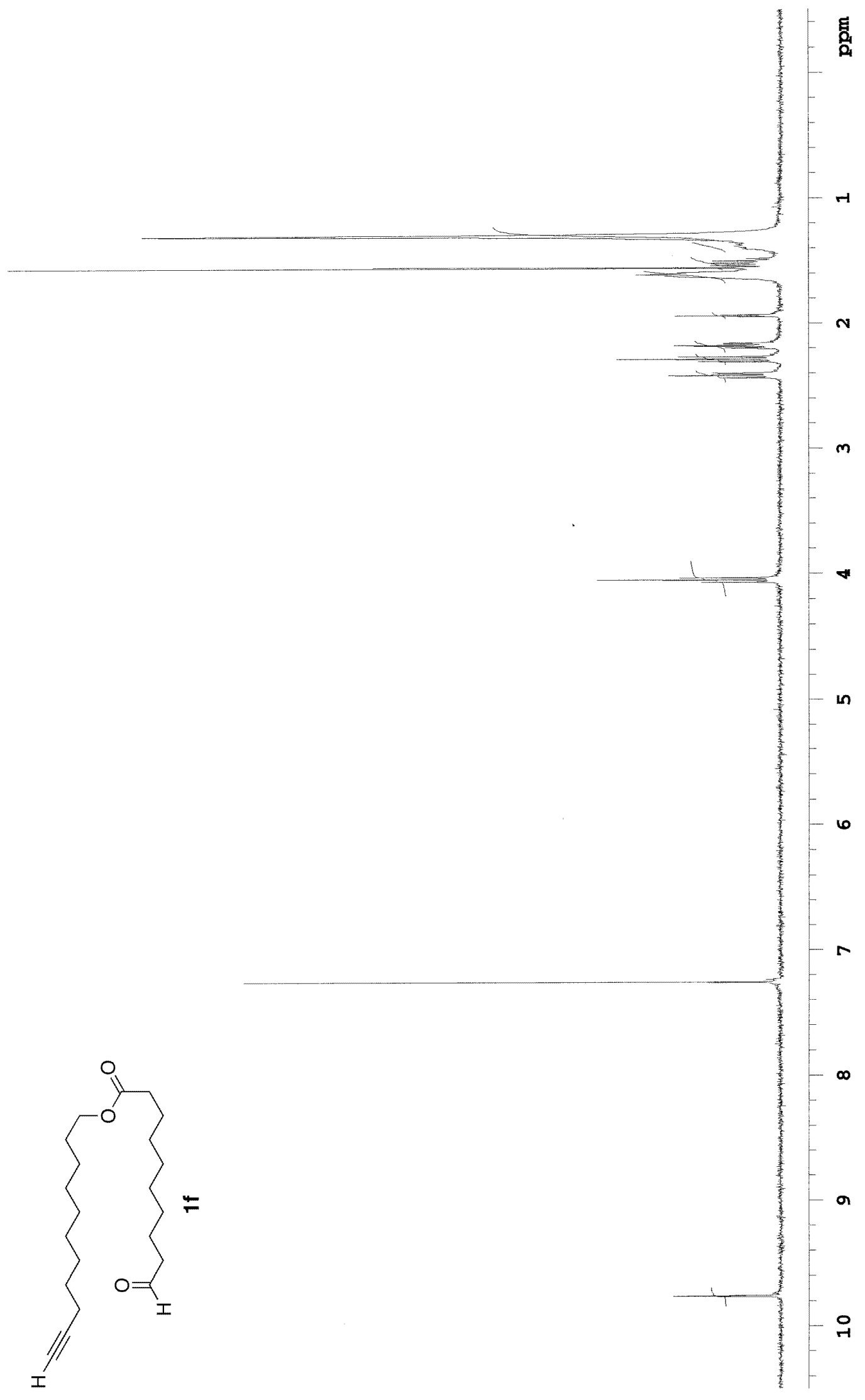




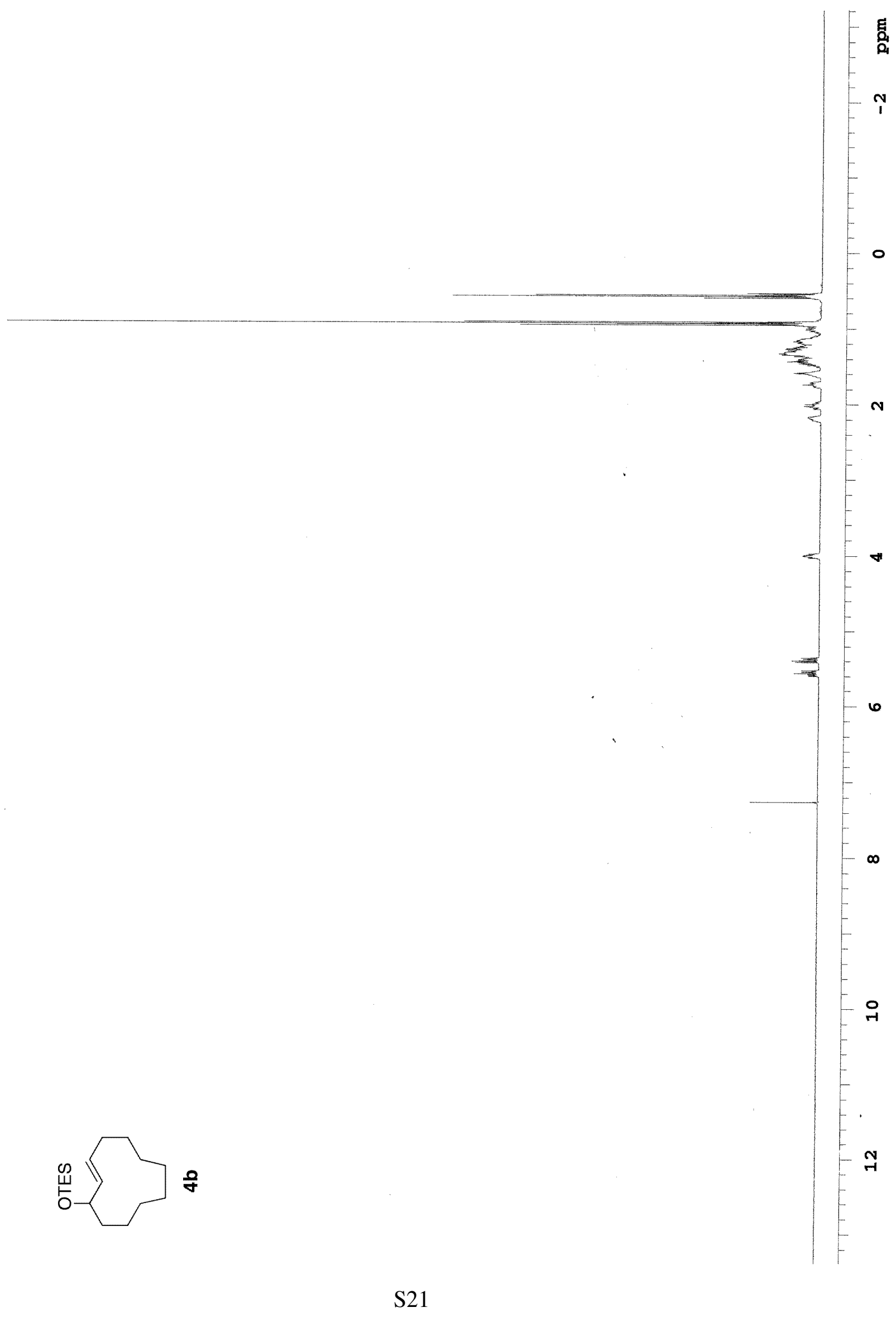




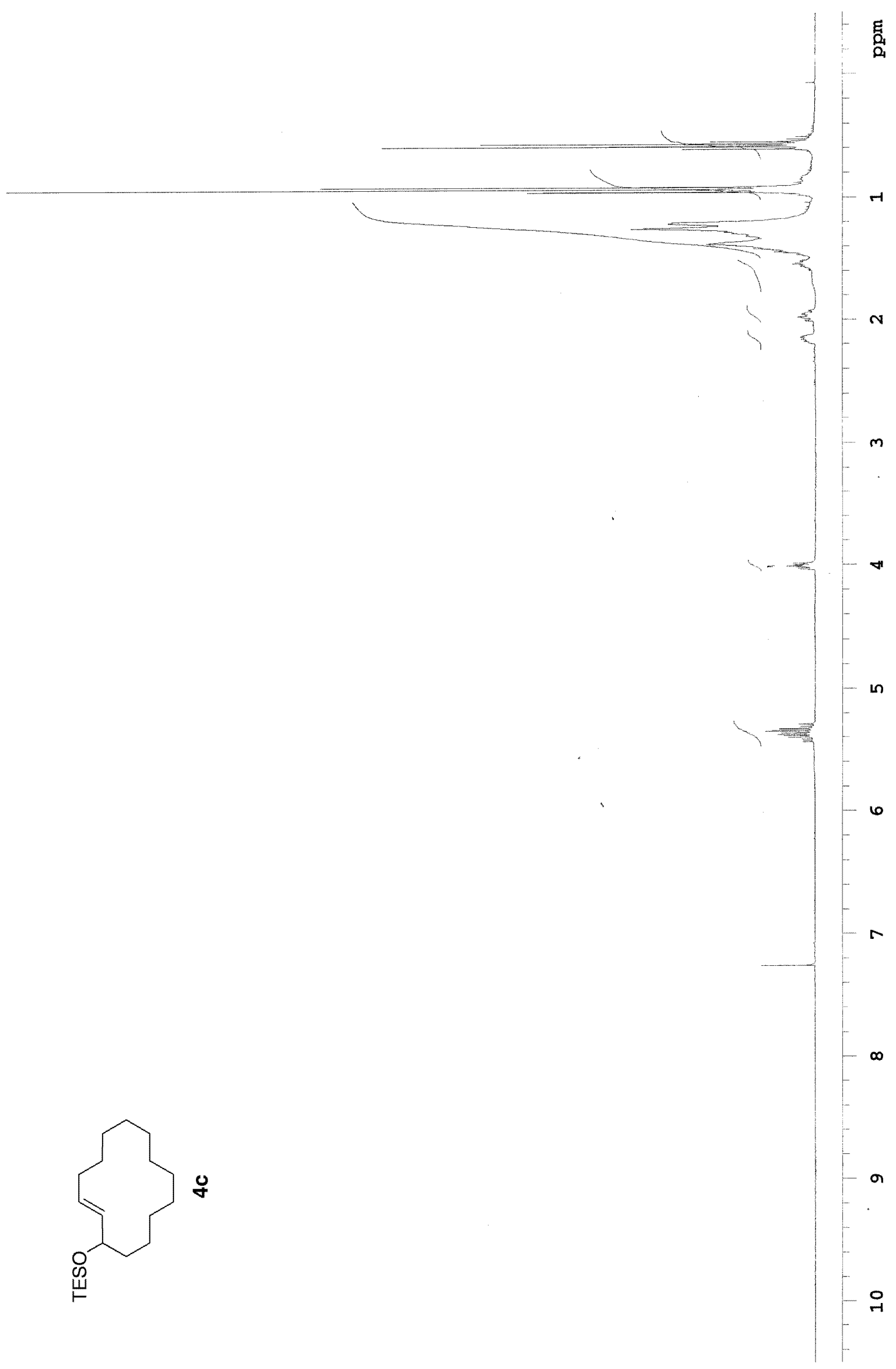




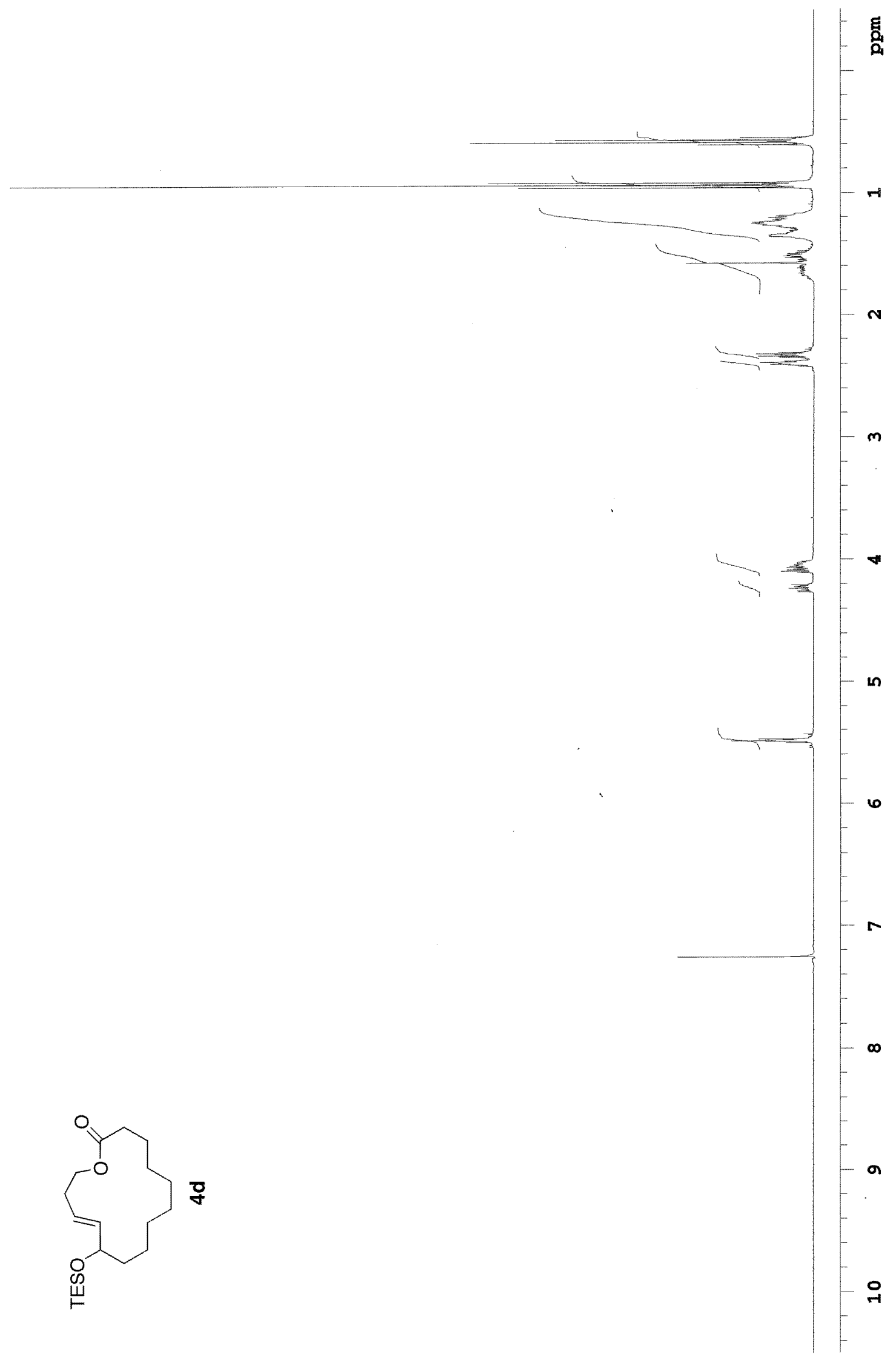




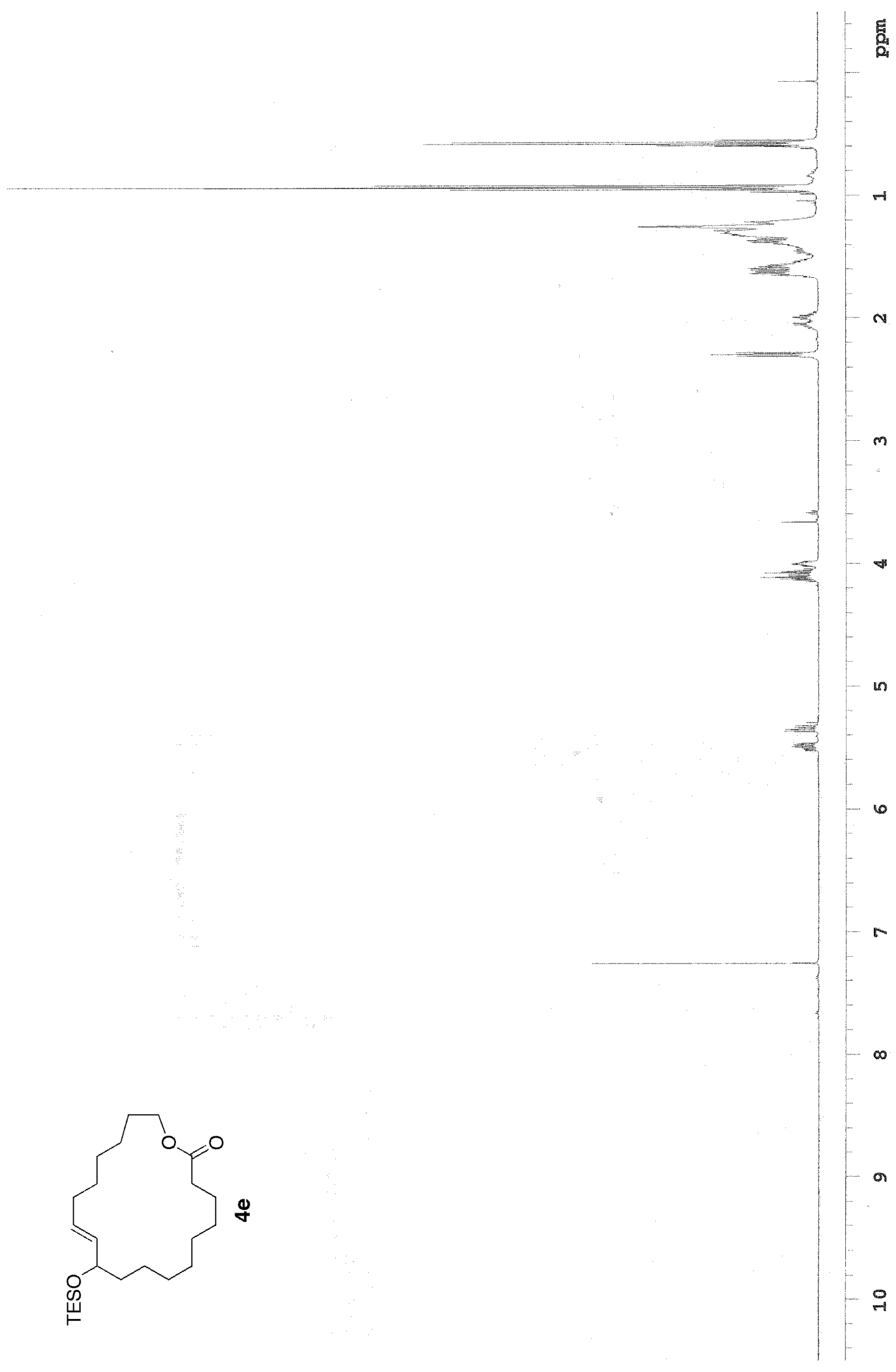




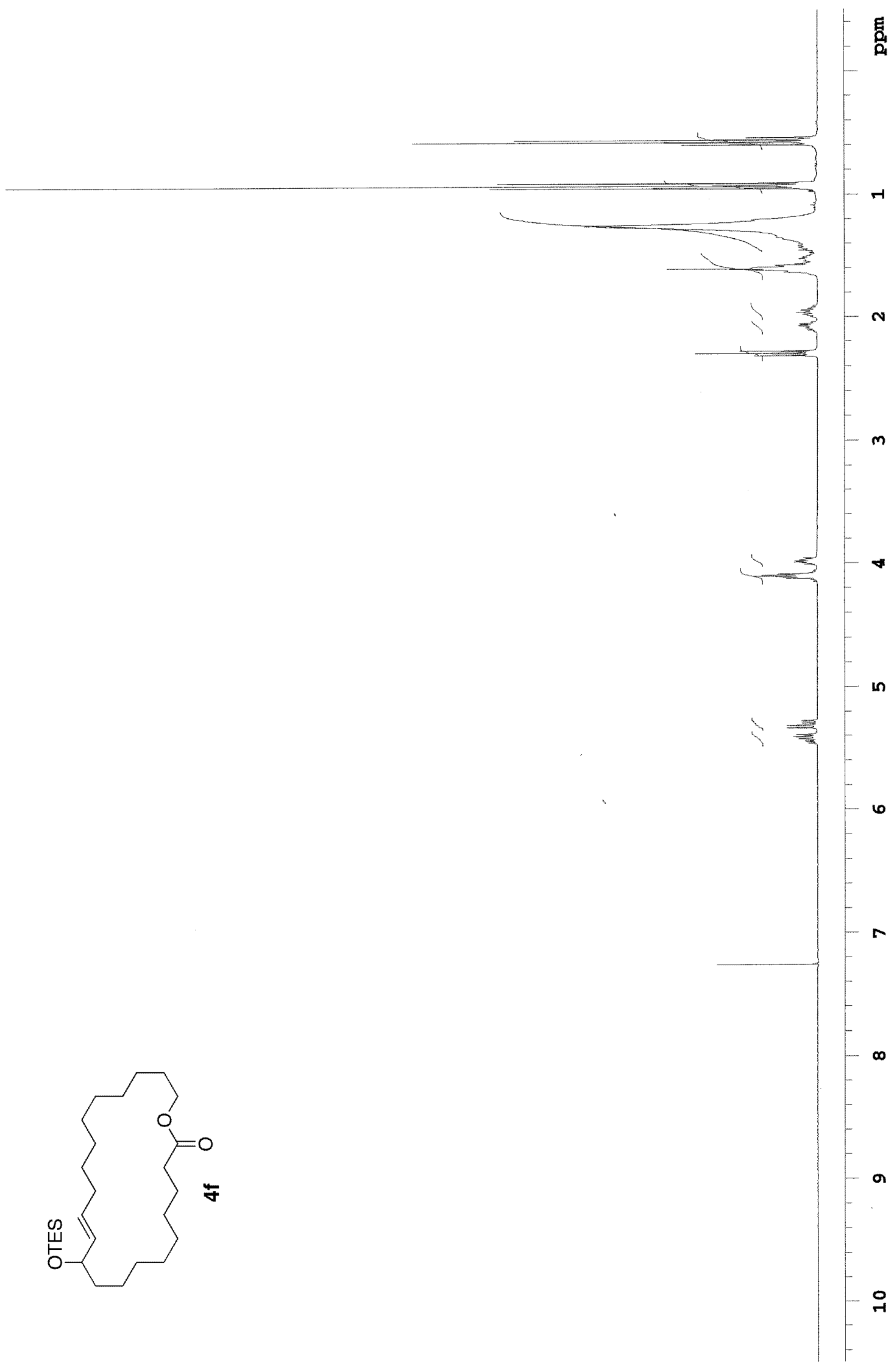




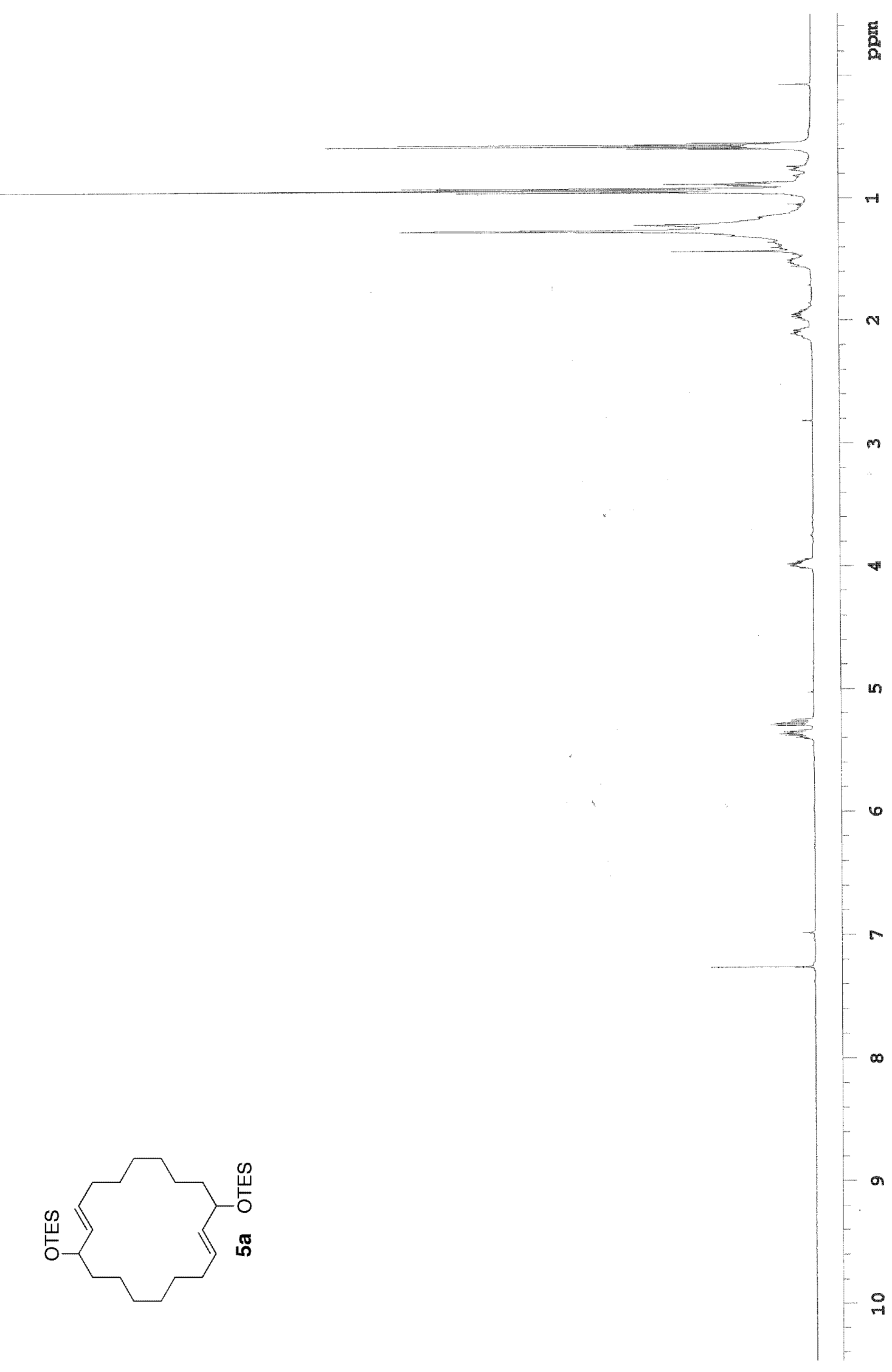




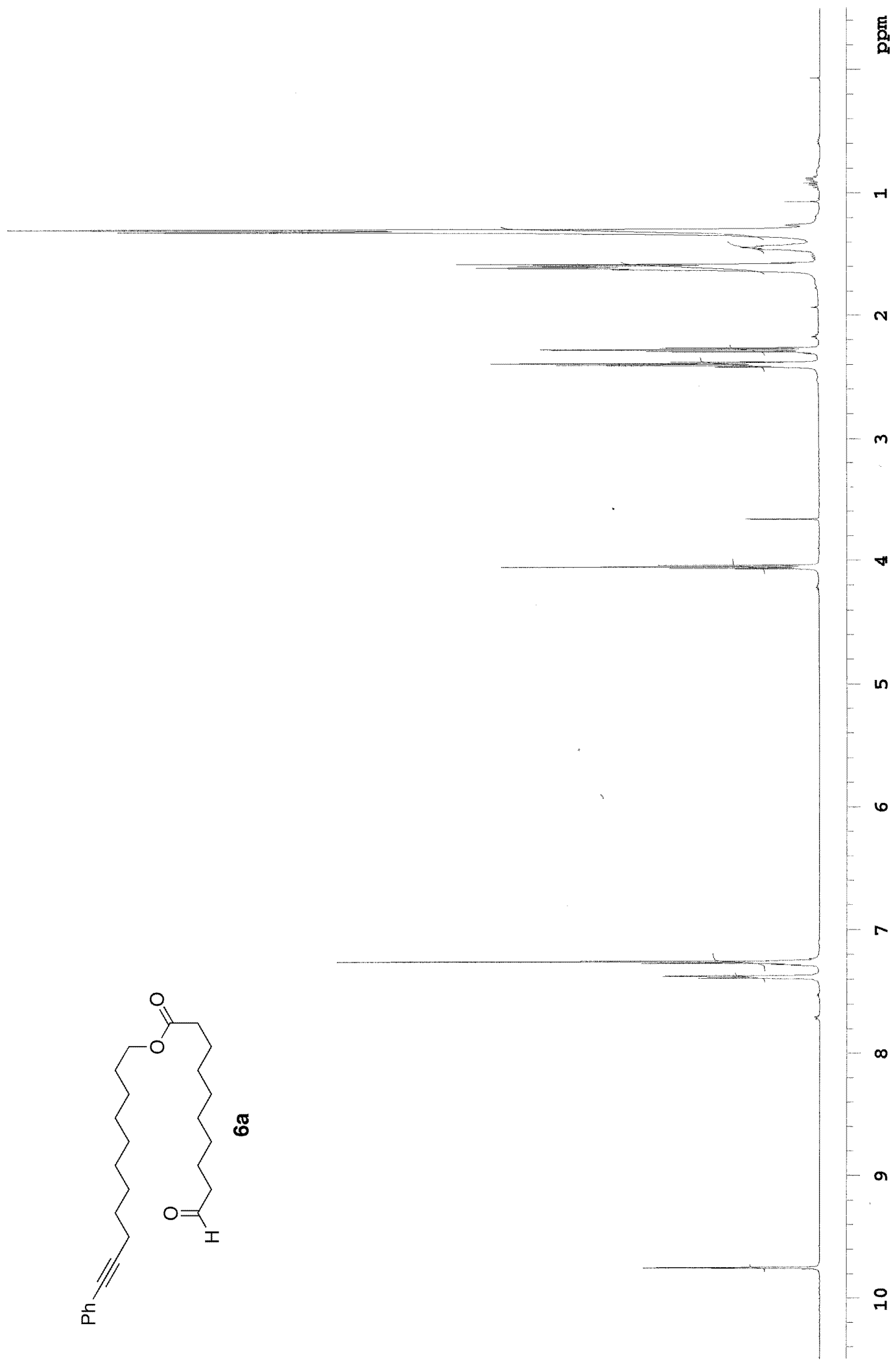




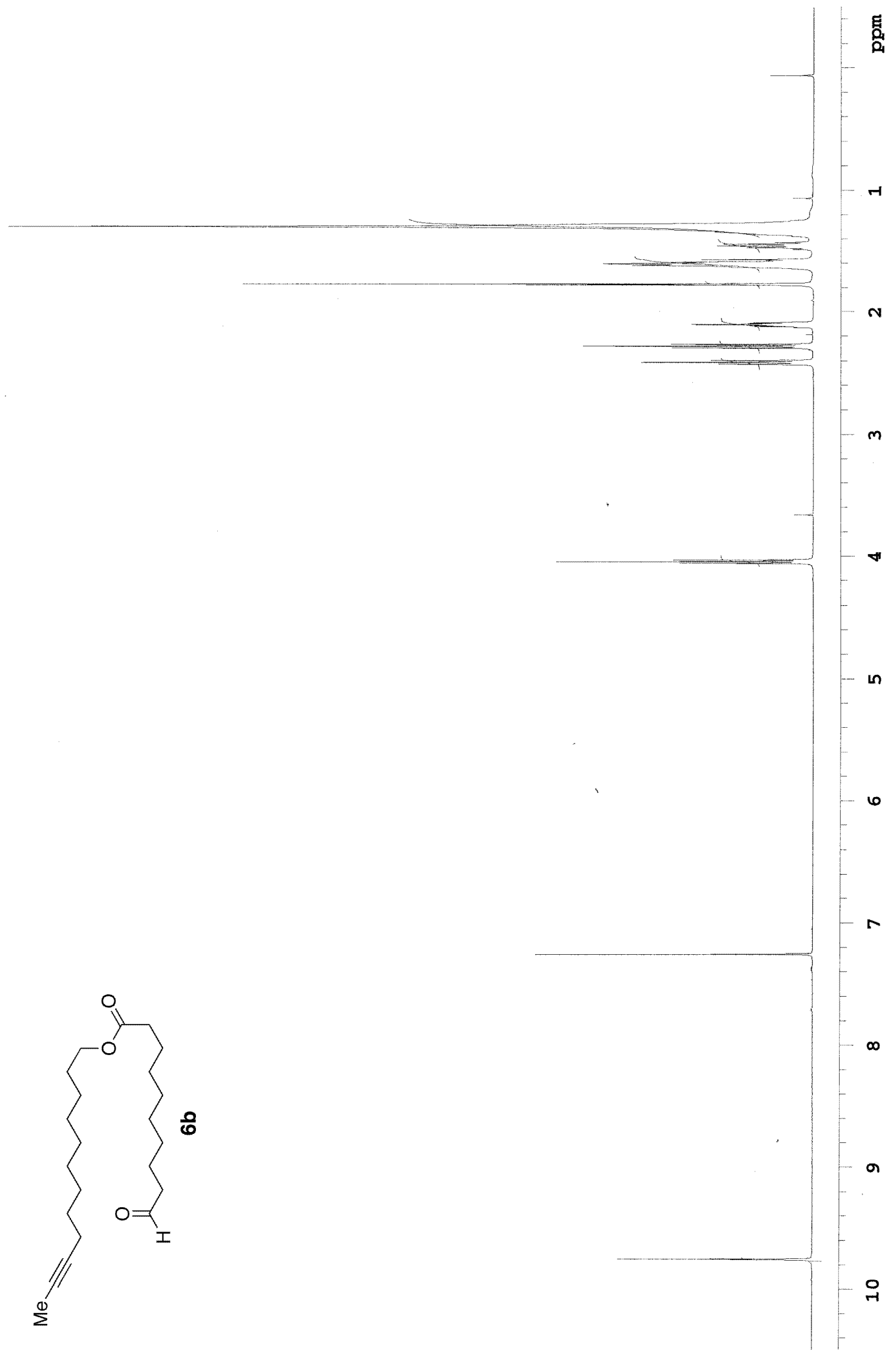




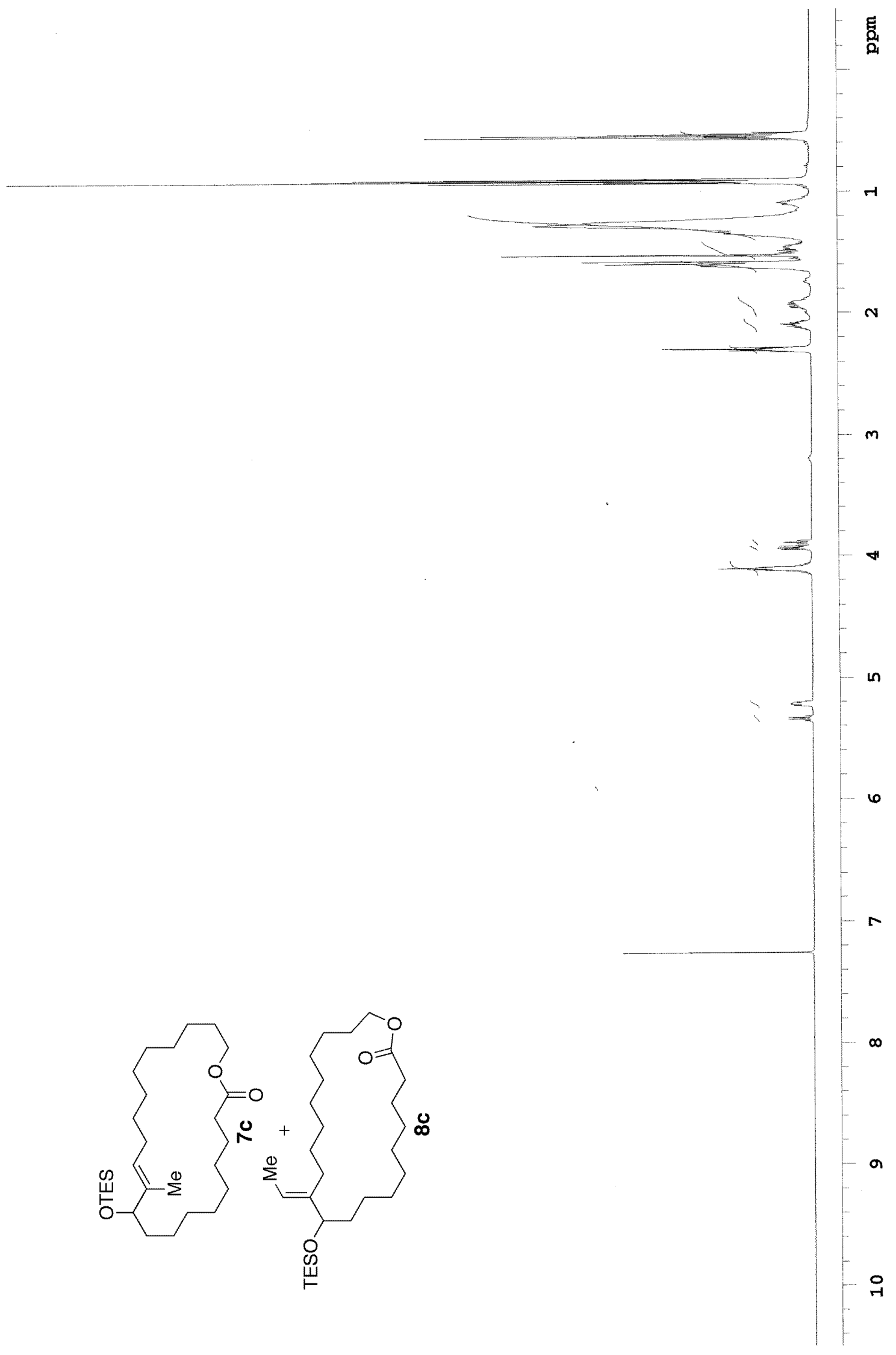




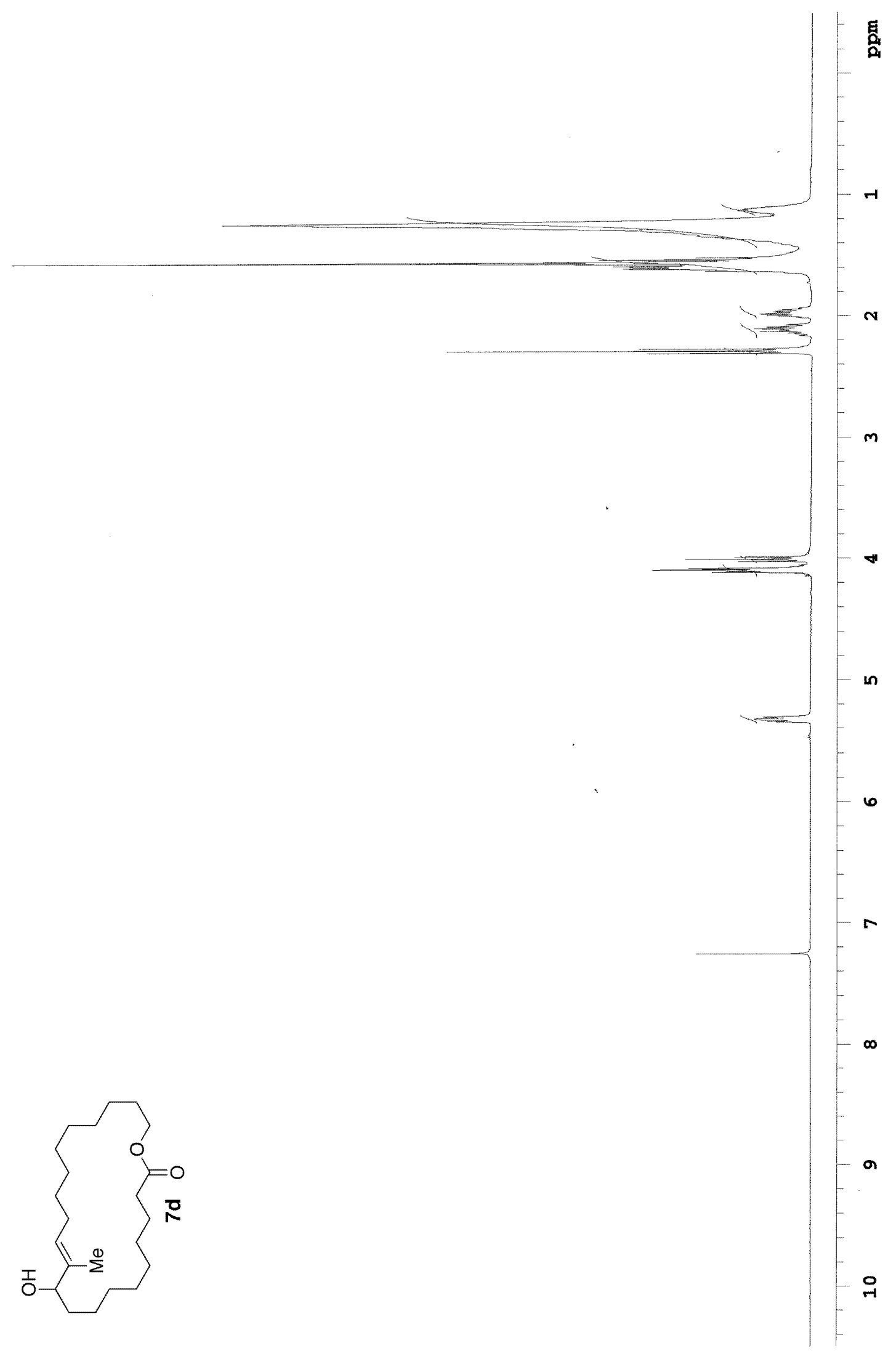




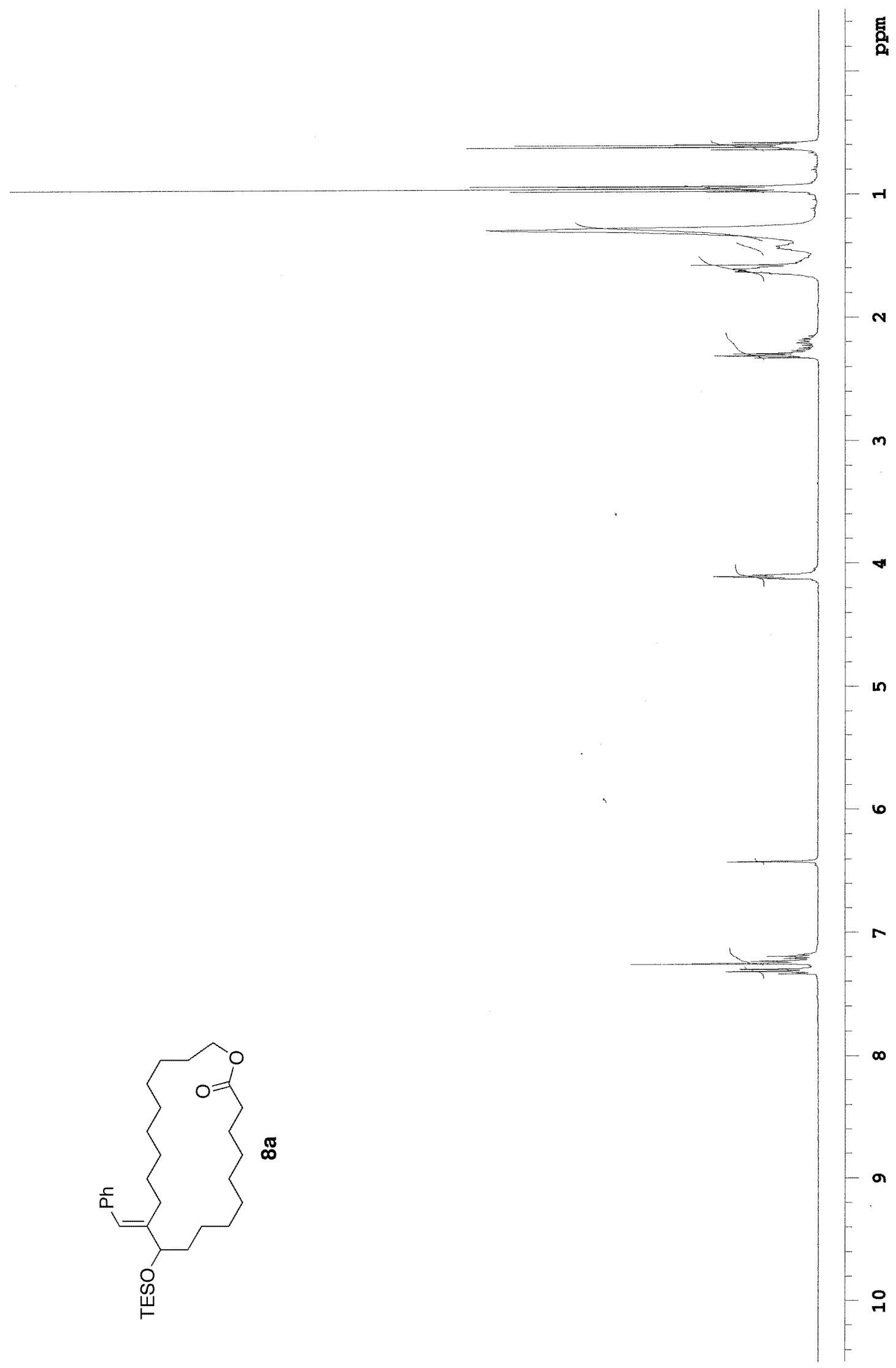




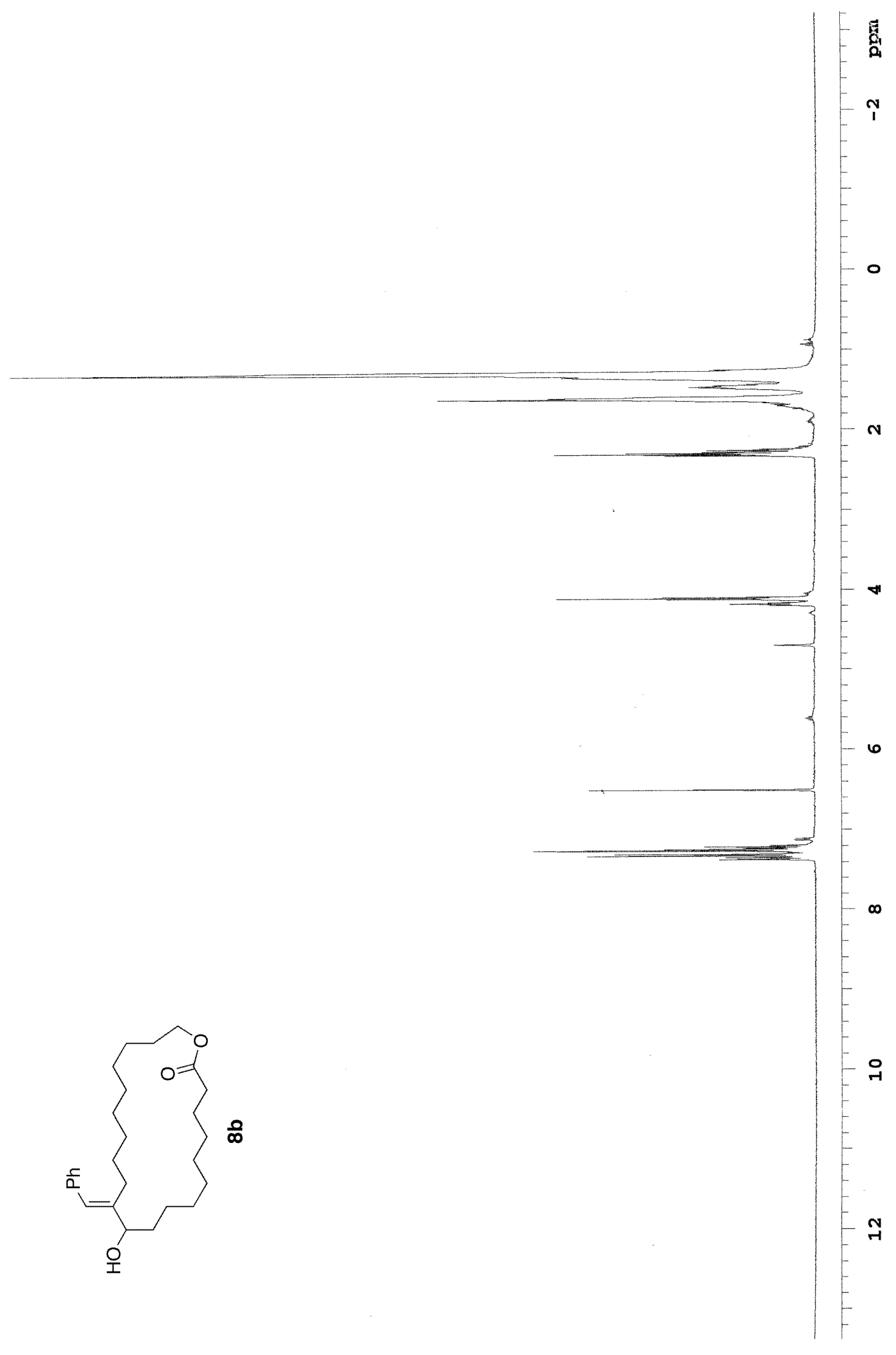




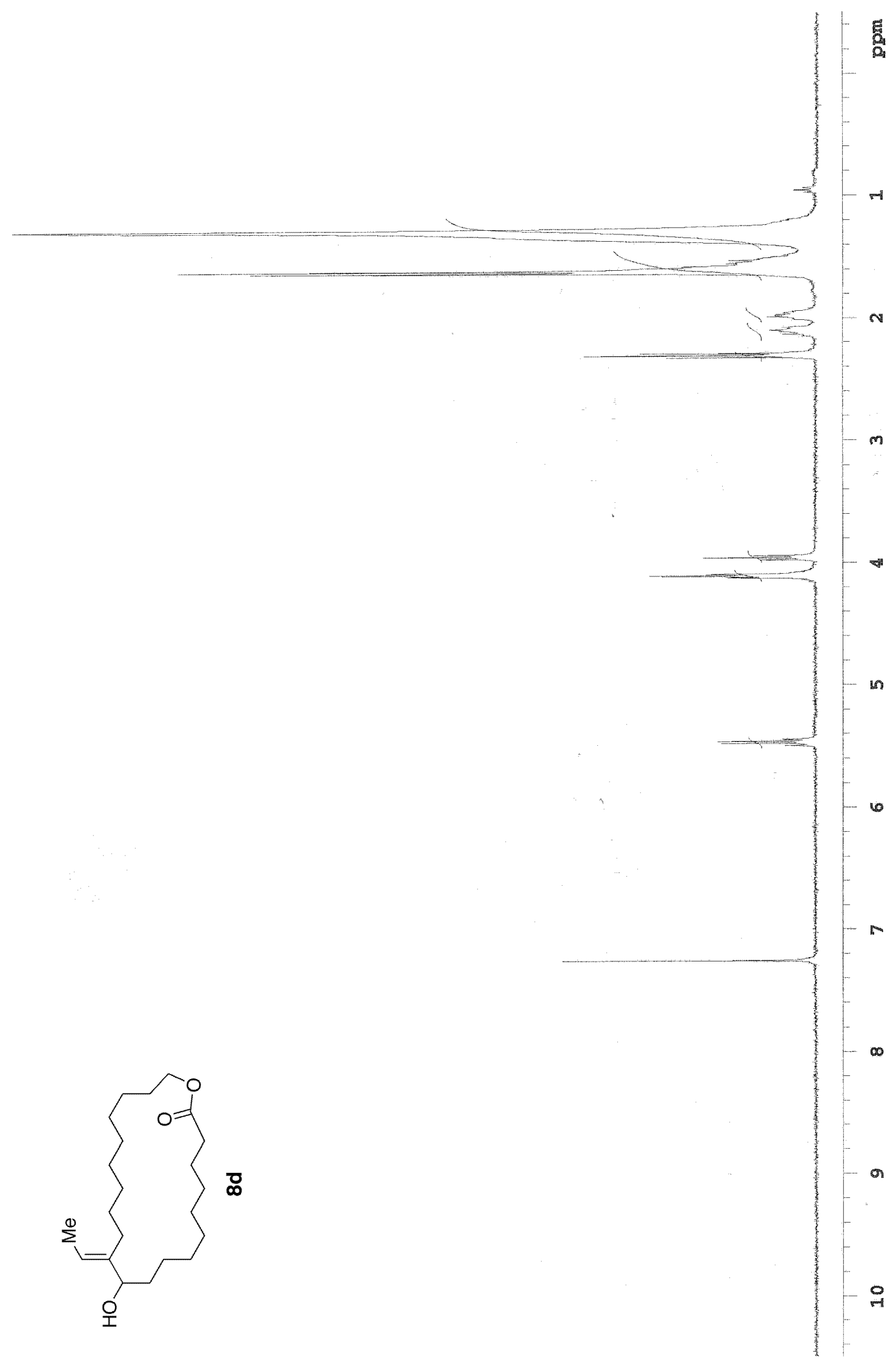




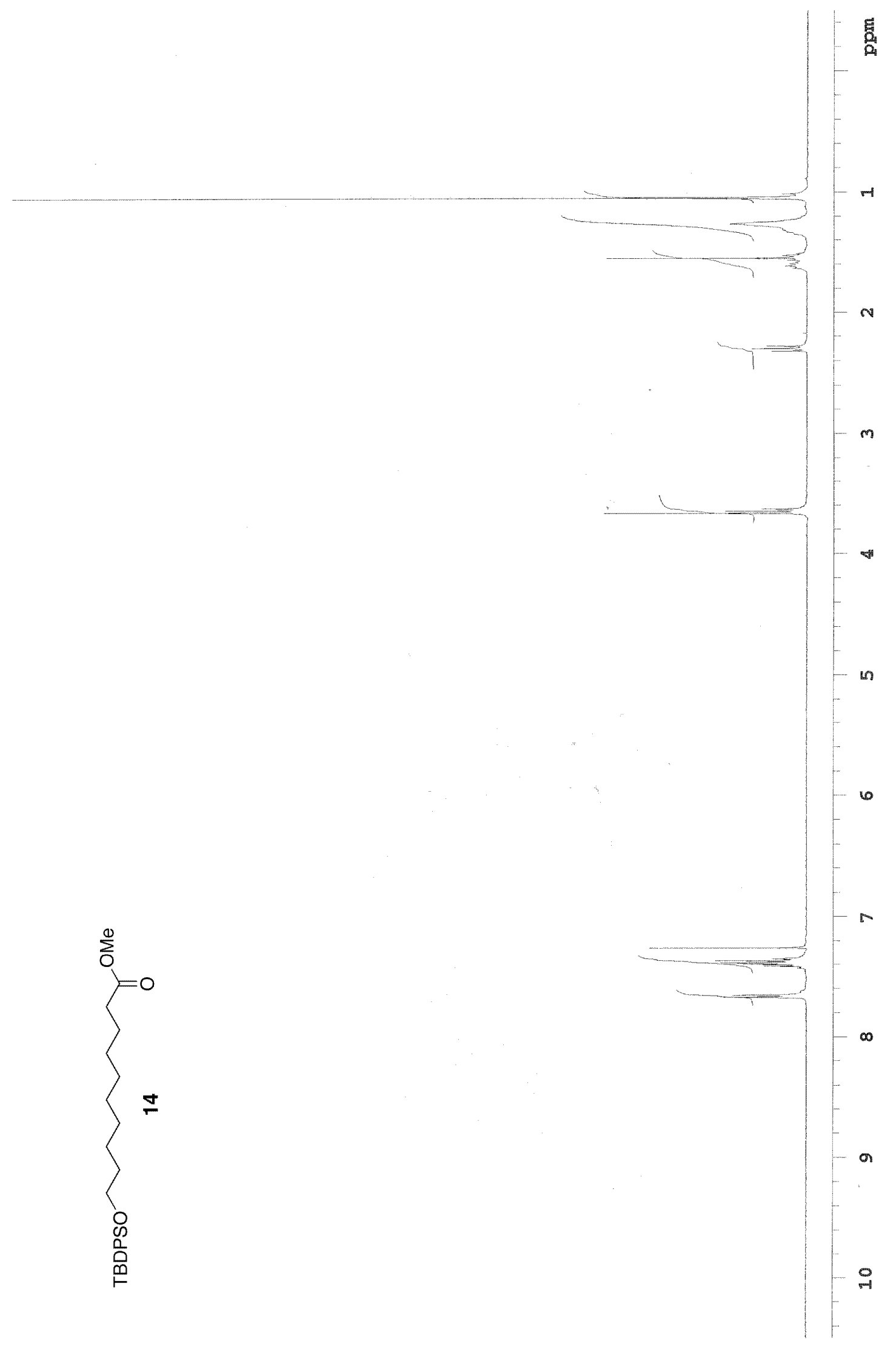




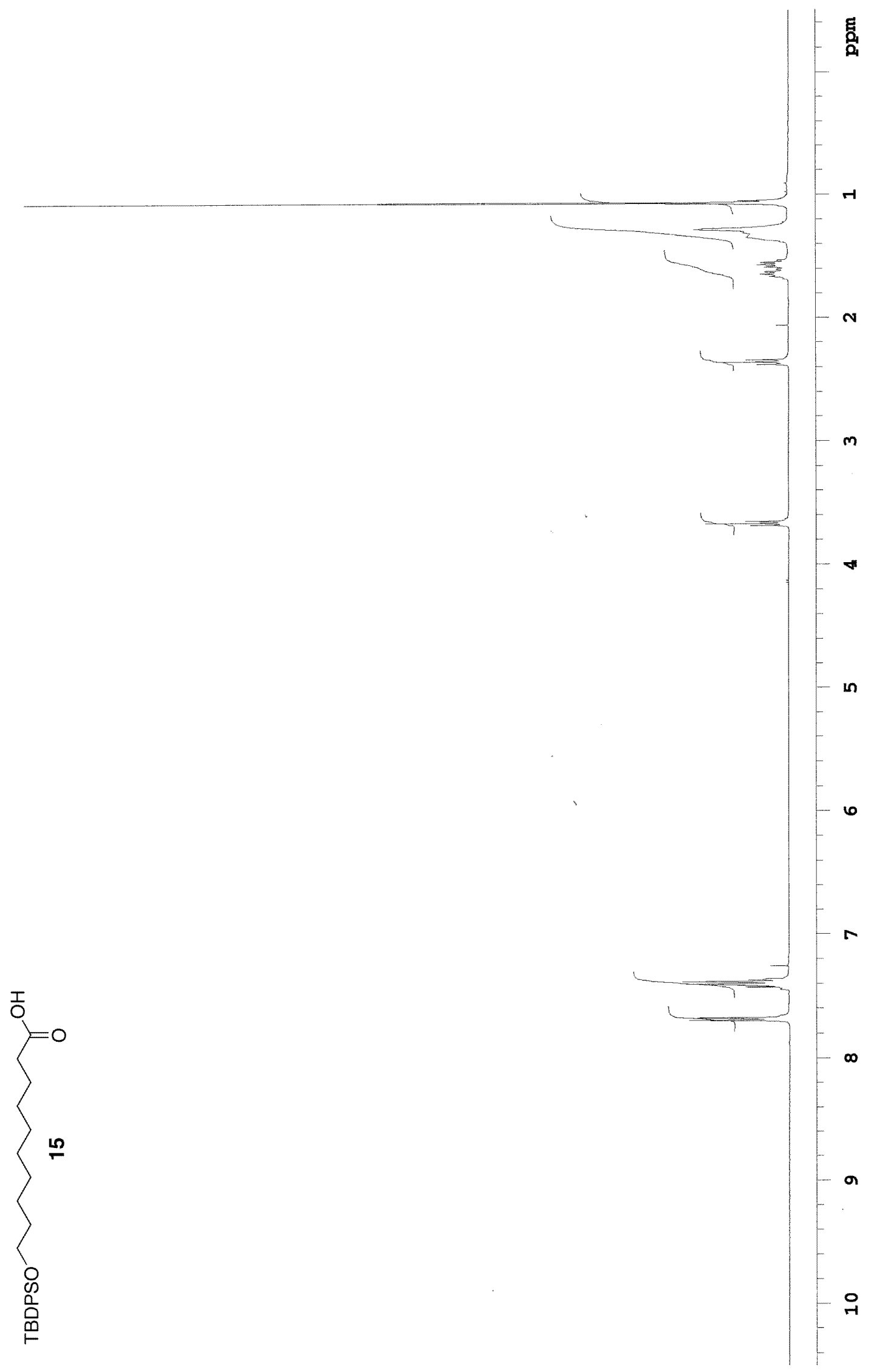




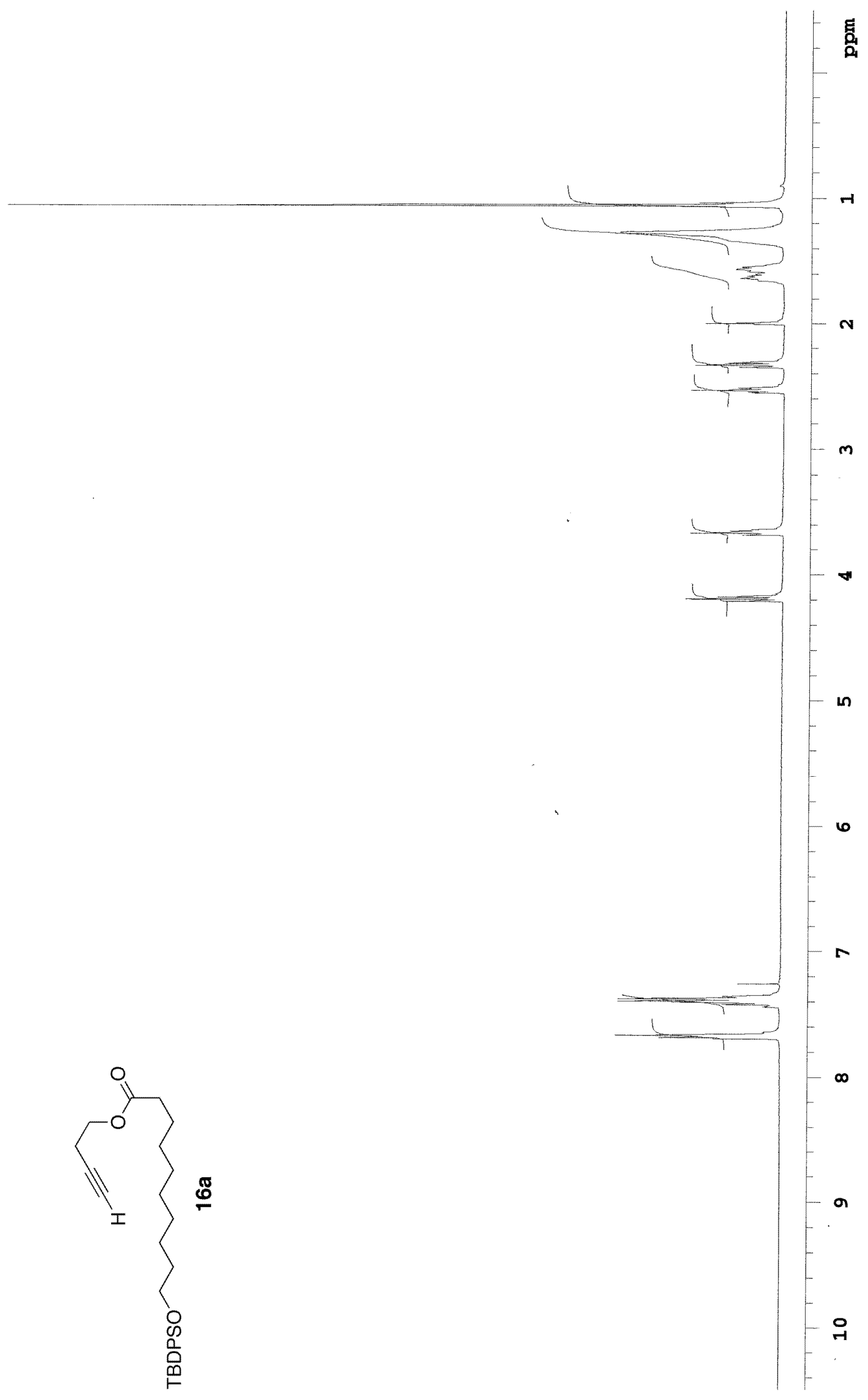




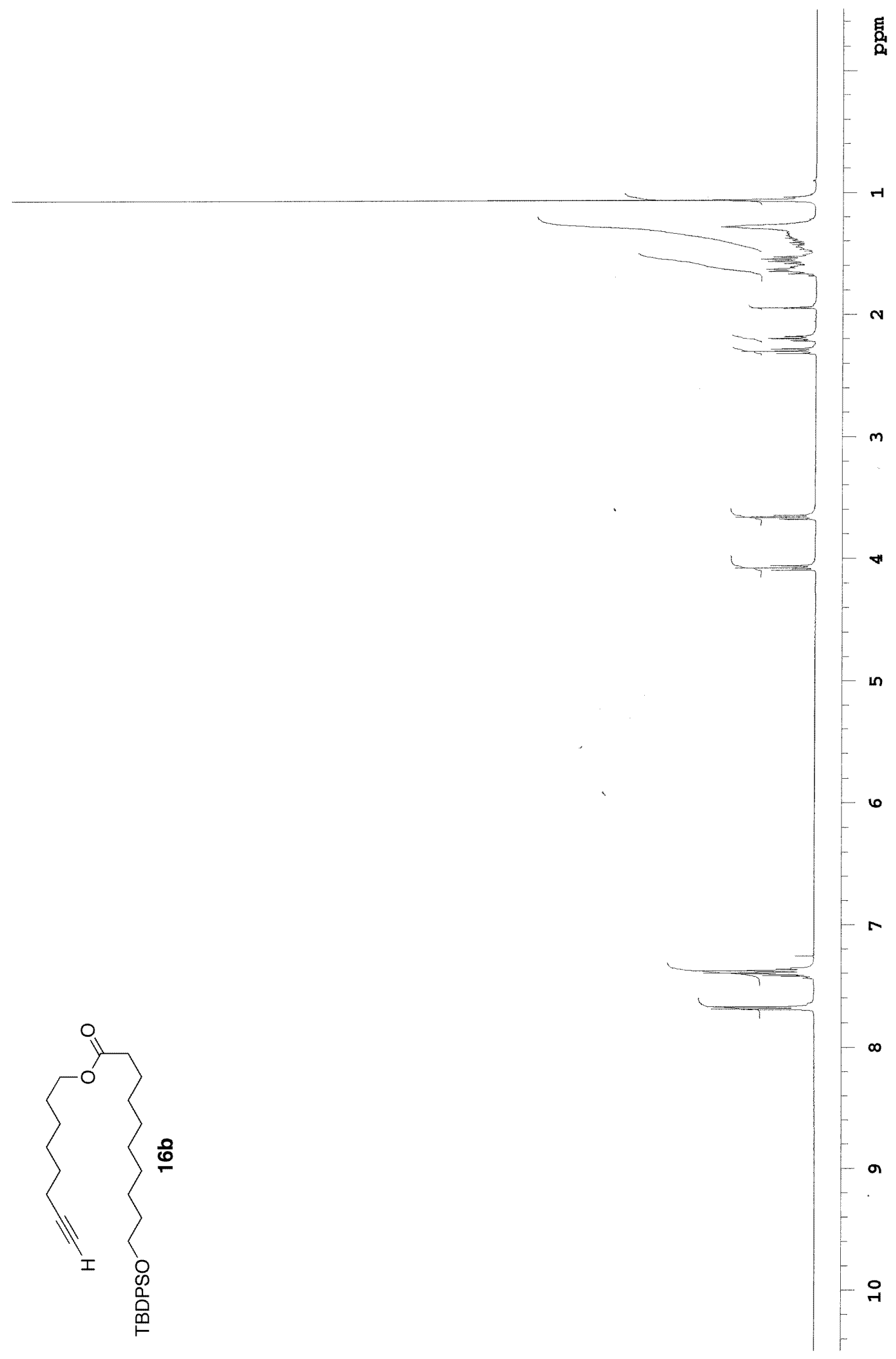




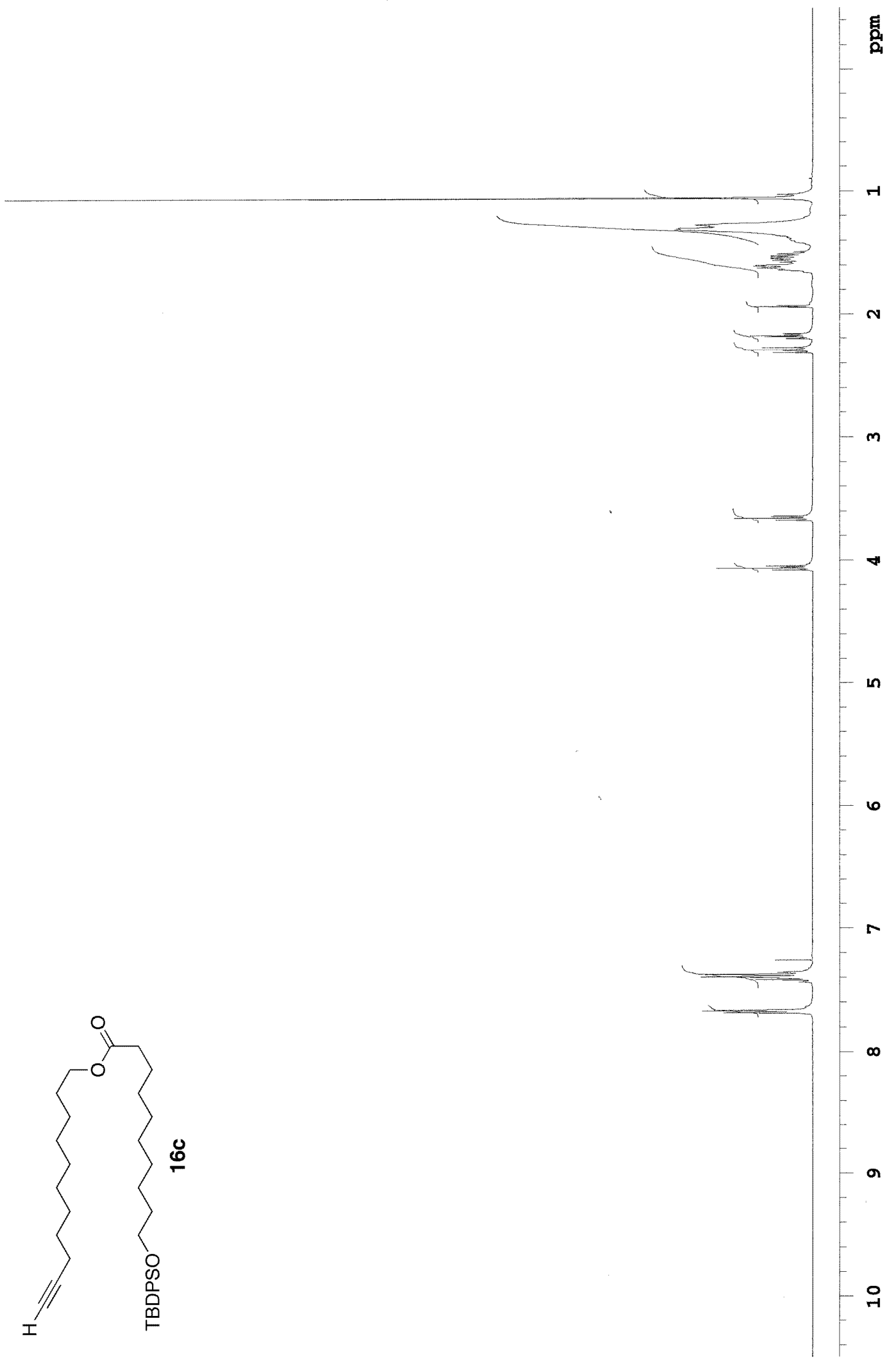




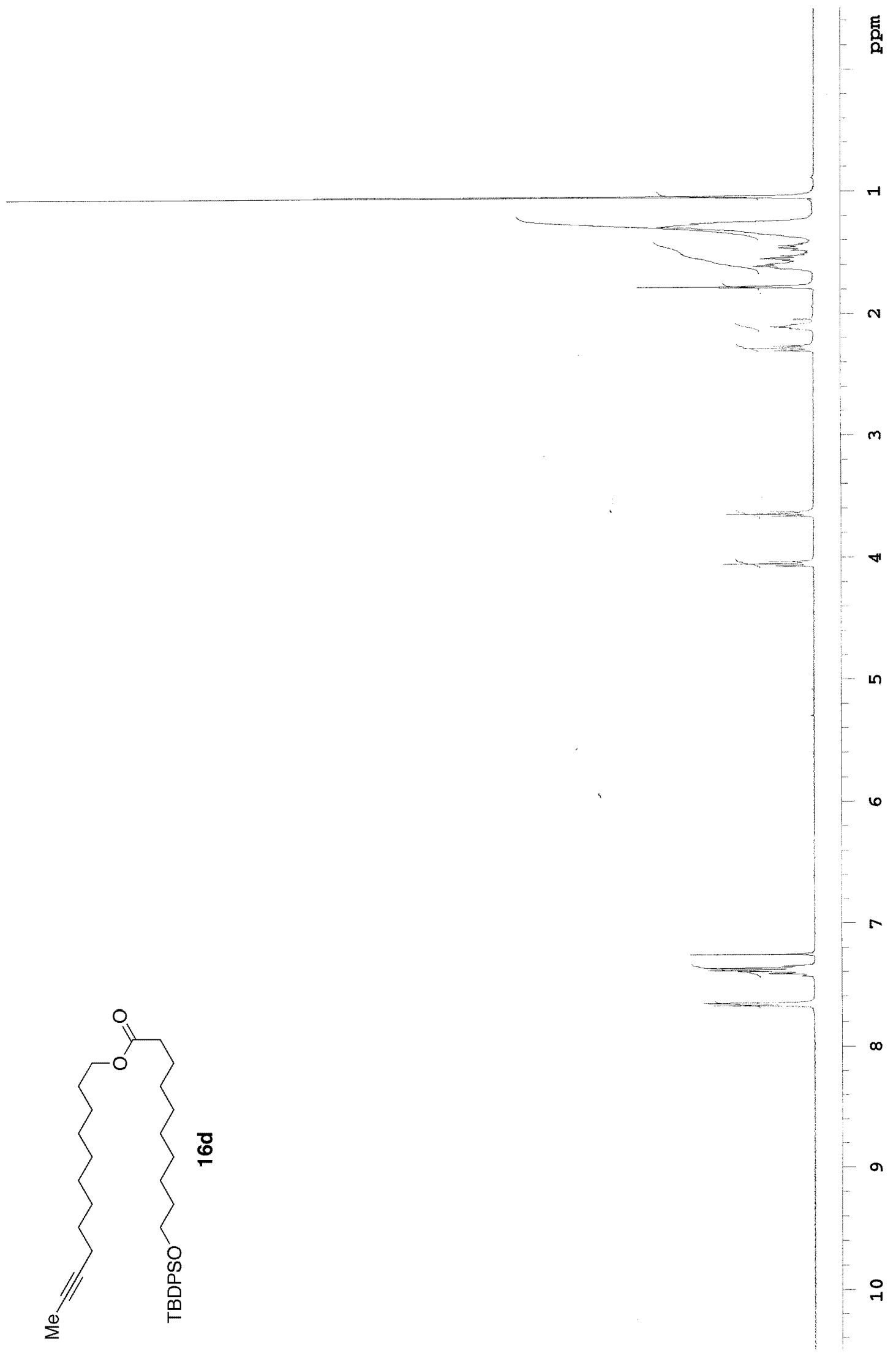




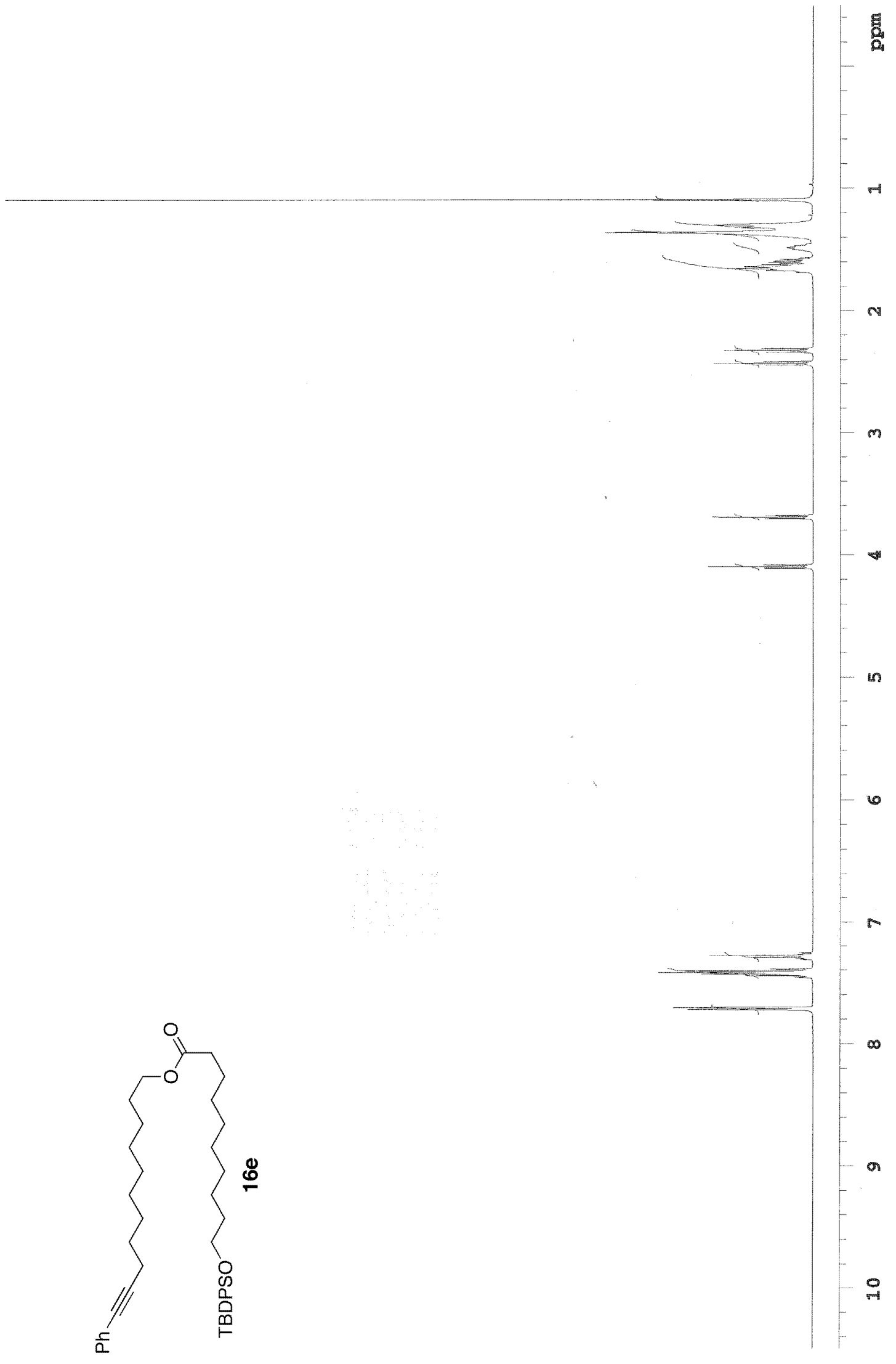




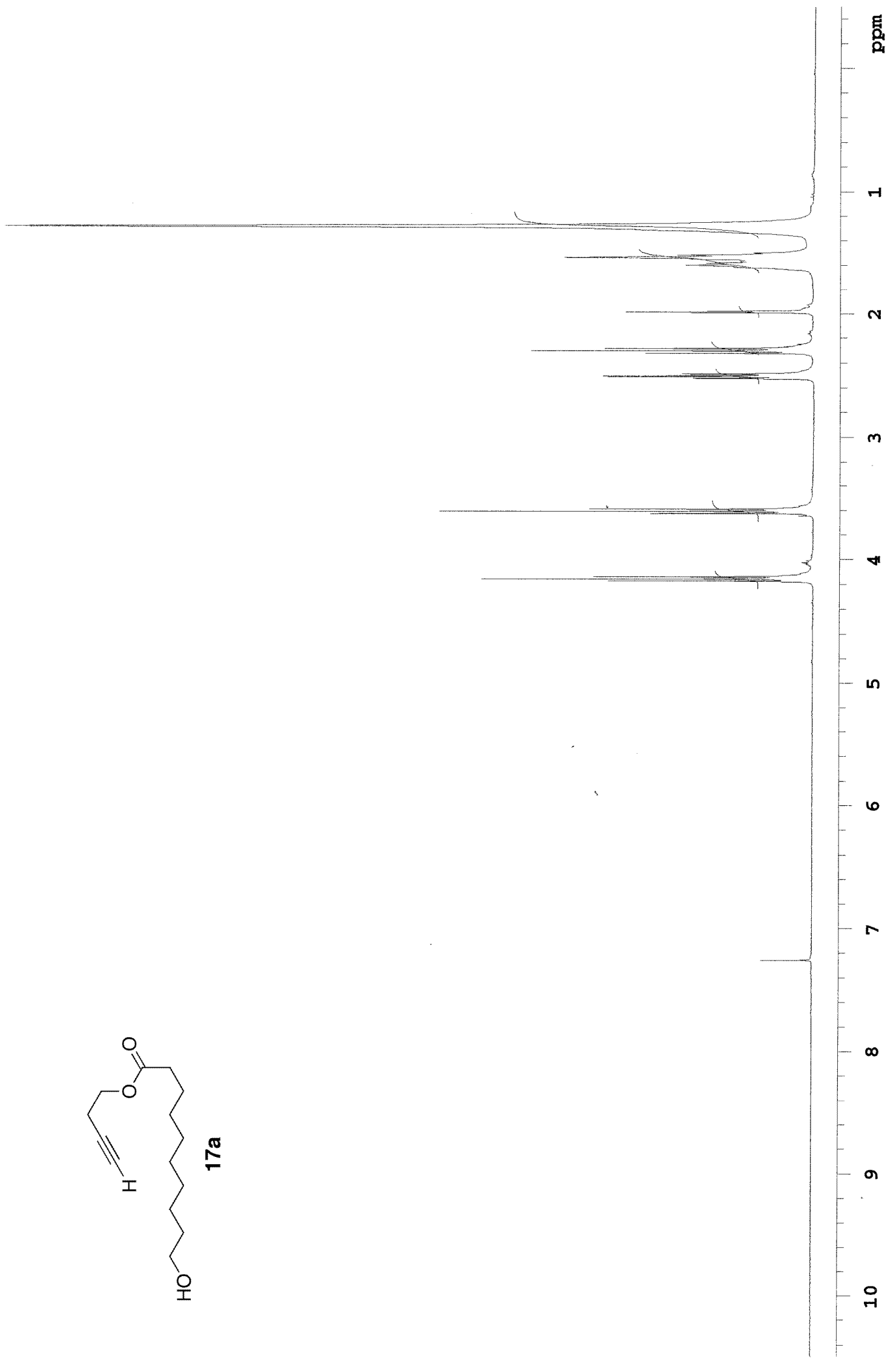




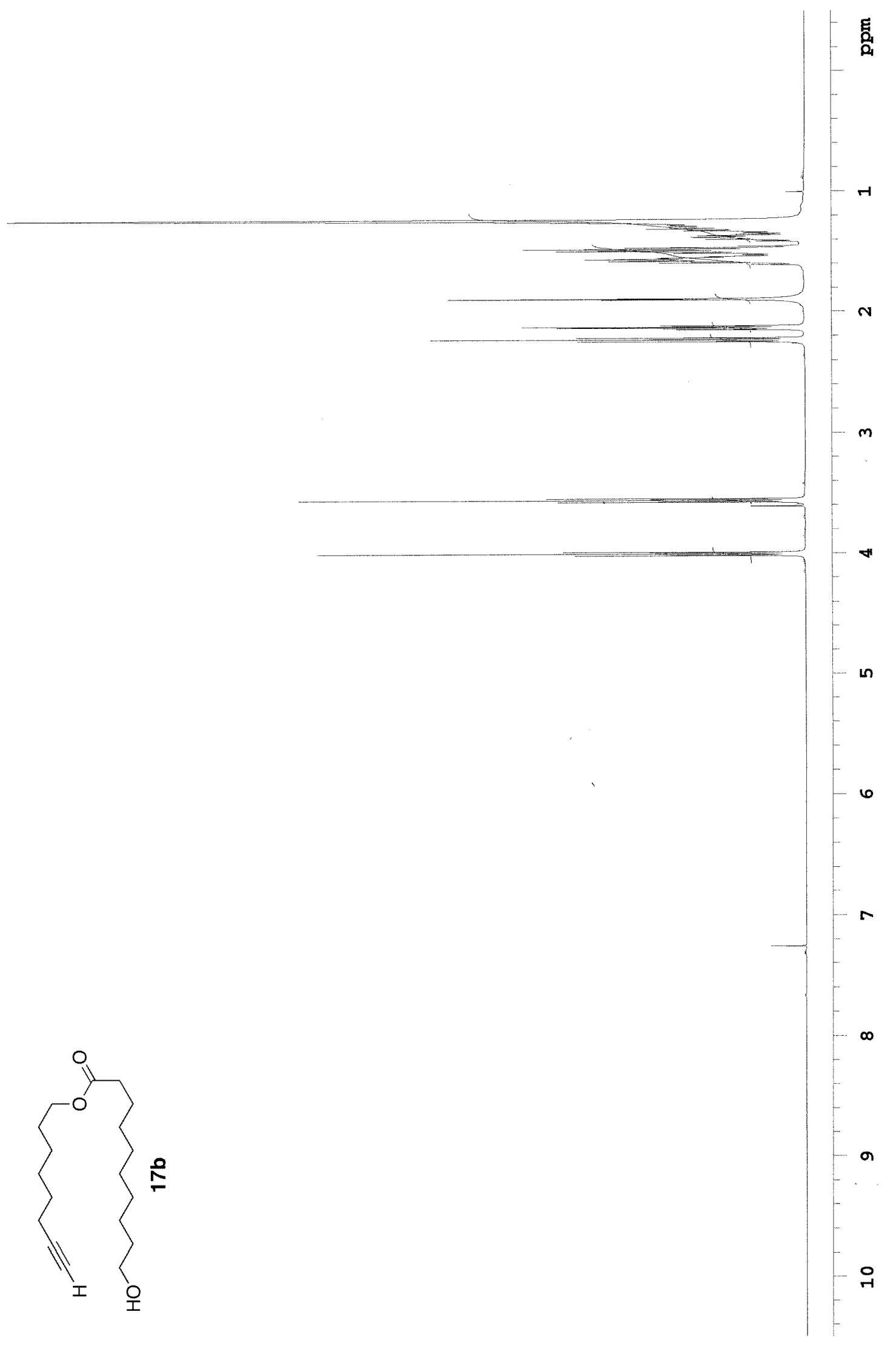




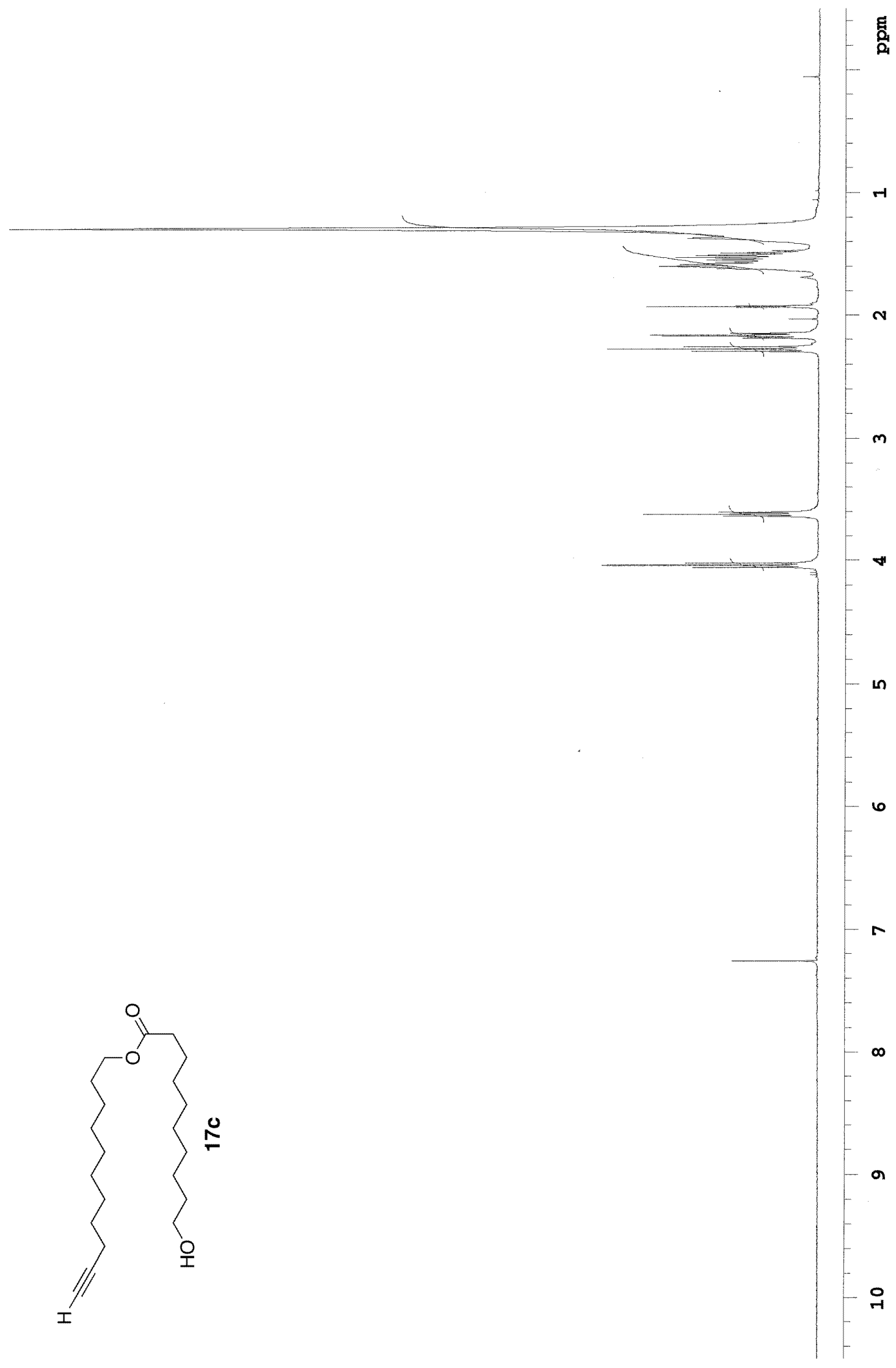




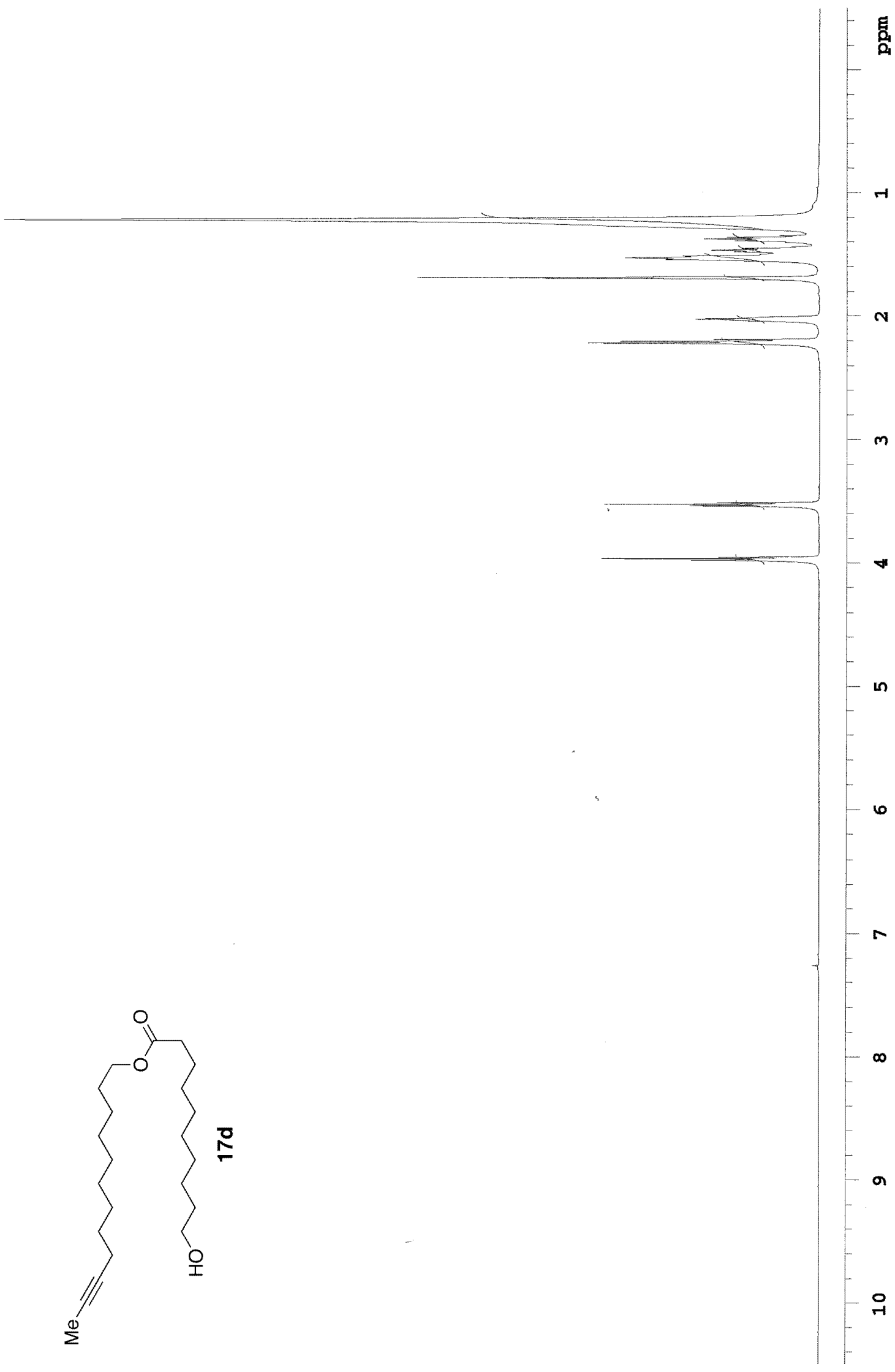




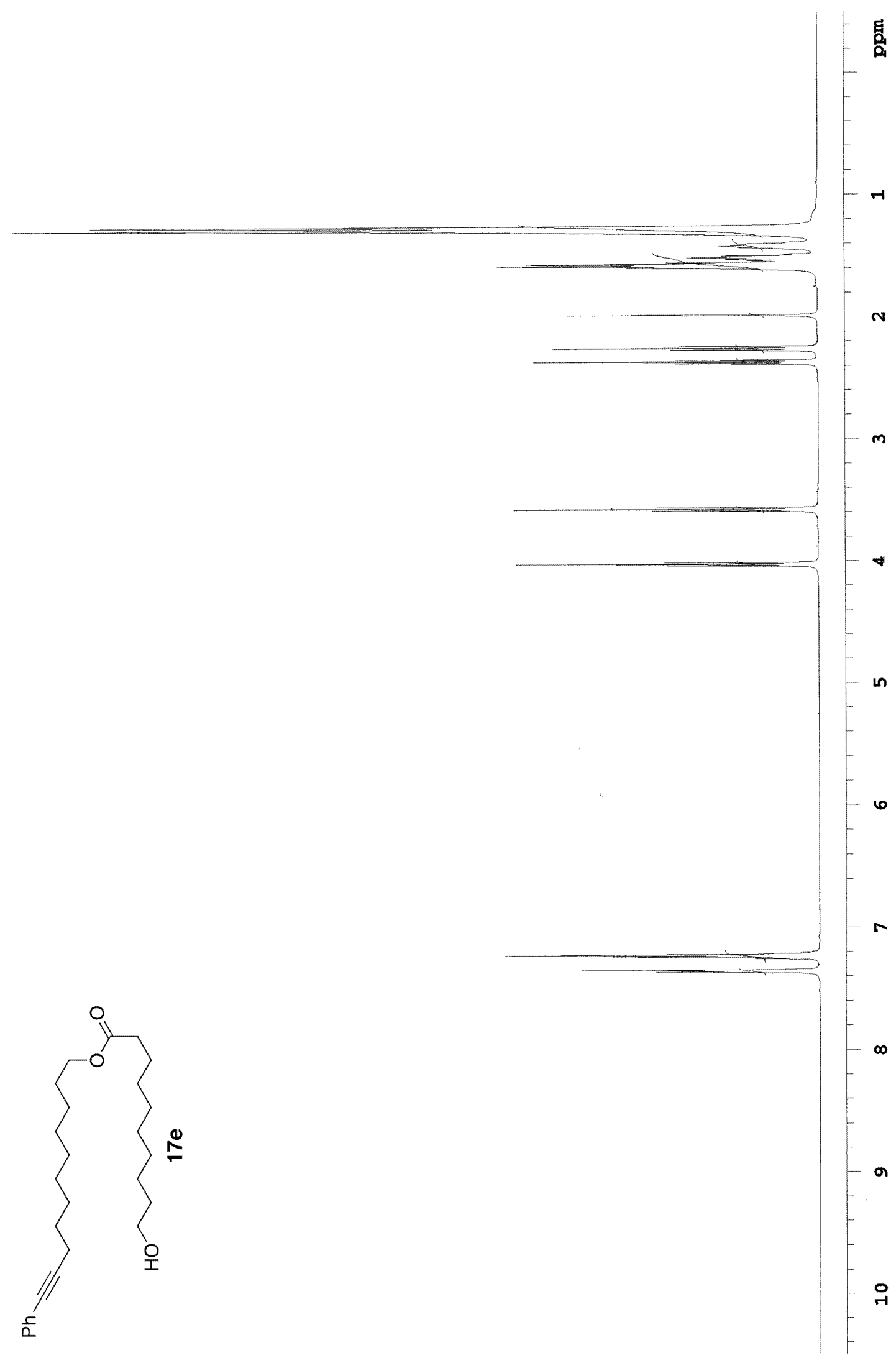

